\title{
Existence criteria via $\alpha-\psi$-contractive mappings of $\varphi$-fractional differential nonlocal boundary value problems
}

\author{
Muhammad Qamar lqbal| ${ }^{1 *}$ [D and Azhar Hussain
}

"Correspondence:

qamariqbaljutt@gmail.com

1 Department of Mathematics,

University of Sargodha, 40100

Sargodha, Pakistan

\section{Springer}

\begin{abstract}
In the existing study, we investigate the criteria of existence of solution for relatively new categories of $\varphi$-Caputo fractional differential equations and inclusions problems equipped with nonlocal $\varphi$-integral boundary conditions. In order to achieve the desired goal, we use $\alpha-\psi$-contractive mappings and the theory of approximate endpoint. In the final stage, we exhibit some examples to provide the illustrations of our theoretical findings.
\end{abstract}

Keywords: $\alpha-\psi$-contractions; Endpoint theory; Approximate fixed point

\section{Introduction}

The calculus of arbitrary order, particularly the fractional order calculus, has been considered as the most useful branch of mathematics in various applied sciences and engineering. In order to foster the large capacity of identities and operators introduced in this theory, the scientist and researchers focused on modeling the variety of physical phenomena involving fraction settings. The researchers have made a significant amount of contribution towards the investigation of fractional differential equations (FDEs) and inclusions (FDIs) which can be seen in [1-10] and the references cited therein. Utilizing the techniques of functional analysis and fixed point theory, a huge number of writings by many academicians have appeared, in which the existence aspects, the property of uniqueness, and the stability analysis of solutions for a variety of FDEs and FDIs of initial and boundary value problems are analyzed [11-14].

Almeida [15] in 2017 launched an extension of classical Caputo operator to $\varphi$-Caputo operator, where the kernel relies on the increasing function $\varphi$. The $\varphi$-Caputo fractional derivative's most often used advantage is its ability to gather all previously implemented fractional derivatives. This generalized operator has the semigroup property, which is essential for obtaining the solution structure. Therefore, the derivative $\varphi$-Caputo fractional is considered as a modified form of a derivative of fractional order. By referring to the $\varphi$ Caputo operator and its generalization, there are many studies which we refer the readers to $[16-22]$.

(c) The Author(s) 2021. This article is licensed under a Creative Commons Attribution 4.0 International License, which permits use sharing, adaptation, distribution and reproduction in any medium or format, as long as you give appropriate credit to the original author(s) and the source, provide a link to the Creative Commons licence, and indicate if changes were made. The images or other third party material in this article are included in the article's Creative Commons licence, unless indicated otherwise in a credit line to the material. If material is not included in the article's Creative Commons licence and your intended use is not permitted by statutory regulation or exceeds the permitted use, you will need to obtain permission directly from the copyright holder. To view a copy of this licence, visit http://creativecommons.org/licenses/by/4.0/. 
In year 2014, Tariboon et al. [23] considered the following category of fractional differential equations involving nonlocal fractional integral condition:

$$
\left\{\begin{array}{l}
{ }^{R L} D_{0^{+}}^{\sigma^{*}} \mu^{*}(a)=\hbar\left(a, \mu^{*}(a)\right), \quad a \in[0, M] \\
\mu^{*}(0)=0 \\
\mu^{*}(M)=\sum_{i=1}^{m} \beta_{i}^{\mathcal{H}} I_{0^{+}}^{p_{i}, \varphi} \mu^{*}\left(\xi_{i}\right)
\end{array}\right.
$$

where $1<\sigma^{*} \leq 2,{ }^{R L} D_{0^{+}}^{\sigma^{*}}$ denotes the RL-fractional derivative of order $\sigma^{*},{ }^{\mathcal{H}} I_{0^{+}}^{p_{i}}$ denotes the Hadamard fractional derivative of order $p_{i}>0, \xi_{i} \in(0, M), \hbar:[0, M] \times \mathbb{R} \rightarrow \mathbb{R}$ and $\beta_{i} \in \mathbb{R}$, $i \in\{1,2, \ldots, m\}$ with the property that $\sum_{i=1}^{m} \frac{\beta_{i} \xi_{i} \sigma^{*}-1}{\left(\sigma^{*}-1\right)^{p_{i}}} \neq M^{\sigma^{*}-1}$.

In the subsequent year, Ntouyas et al. [24] studied the following class of RL-fractional differential equations along with nonlocal fractional integral conditions of Hadamard type:

$$
\left\{\begin{array}{l}
{ }^{R L} D_{0^{+}}^{\sigma^{*}} \mu^{*}(a)=F\left(a, \mu^{*}(a)\right), \quad a \in[0, M] \\
\mu^{*}(0)=0 \\
\mu^{*}(M)=\sum_{i=1}^{m} \beta_{i}^{\mathcal{H}} I_{0^{+}}^{p_{i}, \varphi} \mu^{*}\left(\xi_{i}\right),
\end{array}\right.
$$

where $1<\sigma^{*} \leq 2,{ }^{R L} D_{0^{+}}^{\sigma^{*}}$ denotes the RL-fractional derivative of order $\sigma^{*},{ }^{\mathcal{H}} I_{0^{+}}^{p_{i}}$ denotes the Hadamard fractional derivative of order $p_{i}>0, \xi_{i} \in(0, M), \hbar:[0, M] \times \mathbb{R} \rightarrow \mathbb{P}(\mathbb{R})$ and $\beta_{i} \in \mathbb{R}, i=1,2, \ldots, m$, with the property that $\sum_{i=1}^{m} \frac{\beta_{i} \xi_{i} \sigma^{*}-1}{\left(\sigma^{*}-1\right)^{p_{i}}} \neq M^{\sigma^{*}-1}$.

The above theory gives us motivation and leads us to introducing the following class of fractional differential equations involving boundary conditions in the framework of $\varphi$ Caputo fractional derivative:

$$
\left\{\begin{array}{l}
{ }^{C} D_{0}^{\sigma^{*}, \varphi} \mu^{*}(a)=\hbar\left(a, \mu^{*}(a)\right), \quad a \in J=\left[s_{0}, M\right] \\
\mu^{*}\left(s_{0}\right)=0 \\
\mu^{*}(M)=\sum_{i=1}^{m} \beta_{i}{ }^{R L} I_{0}^{p_{i}, \varphi} \mu^{*}\left(\xi_{i}\right),
\end{array}\right.
$$

where ${ }^{C} D_{0}^{\sigma^{*}, \varphi}$ is the $\varphi$-Caputo fractional derivative of order $\sigma^{*}, 1<\sigma^{*}<2,{ }^{R L} I_{0}^{p_{i}, \varphi}$ is the RL$\varphi$-fractional integral of order $p_{i}>0, \xi_{i} \in\left(s_{0}, M\right), \hbar: J \times \mathbb{R} \rightarrow \mathbb{R}$ and $\beta_{i} \in \mathbb{R} i=1,2, \ldots, m$. We also explore the solutions' existence of the following fractional differential inclusion BVP:

$$
\left\{\begin{array}{l}
{ }^{C} D_{0}^{\sigma^{*}, \varphi} \mu^{*}(a) \in K\left(a, \mu^{*}(a)\right), \quad a \in J \\
\mu^{*}\left(s_{0}\right)=0 \\
\mu^{*}(M)=\sum_{i=1}^{m} \beta_{i}{ }^{R L} I_{0}^{p_{i}, \varphi} \mu^{*}\left(\xi_{i}\right)
\end{array}\right.
$$

where $K: J \times \mathbb{R} \rightarrow \mathbb{P}(\mathbb{R})$ is a set-valued compact map.

By reviewing a broad variety of published papers concerning existence and notions of uniqueness in the light of problems with fractional boundary values, we will see that many authors use conventional methods based on renowned fixed point methods for obtaining desirable outcomes in respect to solutions. Our work in the present research is novel in introducing a new development of two $\varphi$-fractional differential problems equipped with nonlocal boundary conditions and then constructing new operators which belong to a 
new class of core functions. The $\alpha$-admissible and $\alpha-\psi$-counteractive maps are two major functions of this category. Using such a broad class of functions defined on space admitting properties $\left(\mathbf{P}_{1}\right)$ and $\left(\mathbf{P}_{2}\right)$, we investigate the existence criteria of two BVPs (1) and (2). Moreover, we derive another condition of the existence of solutions from the (AEP)property for the assumed multifunction and the aid of endpoint notion. It is notable that our procedures for presumed problems (1) and (2) were used in few works, and we apply these approaches to a nonlocal BVP for the first time.

The contents of the present manuscript are organized in the following way. Within the following section, some necessary notions are mounted in the sense of a $\varphi$-calculus. At the beginning of Sect. 3, we supply a lemma that presents the solution of fractional BVP (1) as an integral equation, and after that utilizing the ideas of Samet et al. [25], the criteria of solutions' existence of the aforementioned BVP are provided. While considering the inclusion version of the proposed BVP, Sect. 4 deals with the solutions' existence with the aid of $\alpha-\psi$-contractive functions on multifunctions and AEP-property of problem (2). The last section is devoted to the illustration of results presented in Sects. 3 and 4 in terms of numerical examples.

\section{Preliminaries}

We compile and study some basic and auxiliary concepts in the present section of this study in the context of our analytical approach. For a function $\mu^{*}:[0,+\infty) \rightarrow \mathbb{R}$, we recall the FRL-integral of order $\sigma^{*}>0$ as follows:

$$
{ }^{R L} I_{s_{0}} \mu^{*} \mu^{*}(a)=\int_{s_{0}}^{a} \frac{(a-q)^{\sigma^{*}-1}}{\Gamma\left(\sigma^{*}\right)} \mu^{*}(q) \mathrm{d} q
$$

for a finite value of the integral $[26,27]$. At this stage, we presume that $n-1<\sigma^{*}<n$ so that $n=\left[\sigma^{*}\right]+1$. The FRL-derivative of order $\sigma^{*}$ for a continuous function $\mu^{*}:[0,+\infty) \rightarrow \mathbb{R}$ is defined by

$$
{ }^{R L} D_{s_{0}}^{\sigma^{*}} \mu^{*}(a)=\left(\frac{\mathrm{d}}{\mathrm{d} a}\right)^{n} \int_{s_{0}}^{a} \frac{(a-q)^{n-\sigma^{*}-1}}{\Gamma\left(n-\sigma^{*}\right)} \mu^{*}(q) \mathrm{d} q
$$

for a finite value of the integral $[26,27]$. Assuming $\mu^{*} \in A C_{\mathbb{R}}^{(n)}([0,+\infty))$ (a class of absolutely continuous functions), Caputo fractional derivative is defined by

$$
{ }^{C} D_{s_{0}}^{\sigma^{*}} \mu^{*}(a)=\int_{s_{0}}^{a} \frac{(a-q)^{n-\sigma^{*}-1}}{\Gamma\left(n-\sigma^{*}\right)} \mu^{*(n)}(q) \mathrm{d} q
$$

for a finite value of the integral $[26,27]$.

Now, assume an increasing function $\varphi \in C^{n}(J)$ with $\varphi^{\prime}(a)>0$ for every $s_{0} \leq a \leq M$. Then an integral in the sense of $\varphi$-Riemann-Liouville of a function $\mu^{*}: J \rightarrow \mathbb{R}$ of order $\sigma^{*}$ depending on the given increasing function $\varphi$ is introduced as

$$
{ }^{R L} I_{s_{0}}^{\sigma^{*} ; \varphi} \mu^{*}(a)=\frac{1}{\Gamma\left(\sigma^{*}\right)} \int_{s_{0}}^{a} \varphi^{\prime}(q)(\varphi(a)-\varphi(q))^{\sigma^{*}-1} \mu^{*}(q) \mathrm{d} q
$$

provided that the RHS of (6) is finite-valued [27-29]. Observe that, if $\varphi(a)=a$, the $\varphi$ FRL integral (6) becomes classical FRL-integral (3). The $\sigma^{*}$ ordered $\varphi$-FRL derivative of a 
continuous function $\mu^{*}:[0,+\infty) \rightarrow \mathbb{R}$ is illustrated as

$$
{ }^{R L} D_{s_{0}}^{\sigma^{*} ; \varphi} \mu^{*}(a)=\frac{1}{\Gamma\left(n-\sigma^{*}\right)}\left(\frac{1}{\varphi^{\prime}(a)} \frac{\mathrm{d}}{\mathrm{d} a}\right)^{n} \int_{s_{0}}^{a} \varphi^{\prime}(q)(\varphi(a)-\varphi(q))^{n-\sigma^{*}-1} \mu^{*}(q) \mathrm{d} q
$$

provided that the RHS of (7) is finite-valued [27-29]. Likewise, if $\varphi(a)=a$, it would be apparent that the $\varphi$-FRL derivative (7) becomes classical RL-derivative (4). Motivated by such operators, Almeida proposed the following formula of a new Caputo derivative of $\varphi$-variant:

$$
{ }^{C} D_{s_{0}}^{\sigma^{*} ; \varphi} \mu^{*}(a)=\frac{1}{\Gamma\left(n-\sigma^{*}\right)} \int_{s_{0}}^{a} \varphi^{\prime}(q)(\varphi(a)-\varphi(q))^{n-\sigma^{*}-1}\left(\frac{1}{\varphi^{\prime}(q)} \frac{\mathrm{d}}{\mathrm{d} q}\right)^{n} \mu^{*}(q) \mathrm{d} q
$$

provided that the RHS of (8) is finite-valued [15]. It is noteworthy that if $\varphi(a)=a$, the $\varphi$-Caputo fractional derivative (8) becomes classical Caputo derivative (5). In the following lemmas, we can observe some interesting properties of the $\varphi$-fractional operators of Caputo and Riemann-Liouville sense.

Lemma 2.1 ([15, 27-29]) Suppose that $\sigma^{*}, \varrho^{*}$, and $\beta^{*}$ are positive, an increasing function $\varphi \in C^{n}(J)$ with $\varphi^{\prime}(a)>0$ for any $a \in J$. Then

(i1) ${ }^{R L} I_{s_{0}}^{\sigma^{*} ; \varphi}\left({ }^{R L} I_{s_{0}}^{*^{*} ; \varphi} \mu^{*}\right)(a)=\left({ }^{R L} I_{s_{0}}^{\sigma^{*}+\varrho^{*} ; \varphi} \mu^{*}\right)(a)$,

(i2) ${ }^{R L} I_{s_{0}}^{\sigma^{*} ; \varphi}\left(\varphi(a)-\varphi\left(s_{0}\right)\right)^{\beta^{*}}=\frac{\Gamma\left(\beta^{*}+1\right)}{\Gamma\left(\sigma^{*}+\beta^{*}+1\right)}\left(\varphi(a)-\varphi\left(s_{0}\right)\right)^{\sigma^{*}+\beta^{*}}$,

(i3) ${ }^{C} D_{s_{0}}^{\sigma^{*} ; \varphi}\left(\varphi(a)-\varphi\left(s_{0}\right)\right)^{\beta^{*}}=\frac{\Gamma\left(\beta^{*}+1\right)}{\Gamma\left(\beta^{*}-\sigma^{*}+1\right)}\left(\varphi(a)-\varphi\left(s_{0}\right)\right)^{\beta^{*}-\sigma^{*}} \quad\left(\beta^{*}>\sigma^{*}\right)$,

(i4) ${ }^{R L} D_{s_{0}}^{\sigma^{*} ; \varphi}\left({ }^{R L} I_{s_{0}}^{\varrho^{*} ; \varphi} \mu^{*}\right)(a)=\left({ }^{R L} I_{s_{0}}^{\varrho^{*}-\sigma^{*} ; \varphi} \mu^{*}\right)(a), \quad\left(\sigma^{*}<\varrho^{*}\right)$.

Lemma 2.2 ([15]) Assume $\sigma^{*} \in(n-1, n)$ and $\varphi \in C^{n}(J)$ a nondecreasing function with $\varphi^{\prime}(a)>0$ for every $a \in J$. Then, for any $\mu^{*} \in C^{n-1}(J)$,

$$
{ }^{R L} I_{s_{0}}^{\sigma^{*} ; \varphi}\left({ }^{C} D_{s_{0}}^{\sigma^{*} ; \varphi} \mu^{*}\right)(a)=\mu^{*}(a)-\sum_{j=0}^{n-1} \frac{\left(\delta_{\varphi}\right)^{j} \mu^{*}\left(s_{0}\right)}{j !}\left(\varphi(a)-\varphi\left(s_{0}\right)\right)^{j}, \quad\left(\delta_{\varphi}=\frac{1}{\varphi^{\prime}(a)} \frac{\mathrm{d}}{\mathrm{d} a}\right) .
$$

In light of the aforementioned lemma, the authors have checked that the series solution of the homogeneous equation $\left({ }^{C} D_{s_{0}}^{\sigma^{*} ; \varphi} \mu^{*}\right)(a)=0$ are demonstrated as follows:

$$
\begin{aligned}
\mu^{*}(a) & =\sum_{j=0}^{n-1} \tilde{k}_{j}^{*}\left(\varphi(a)-\varphi\left(s_{0}\right)\right)^{j} \\
& =\tilde{k}_{0}^{*}+\tilde{k}_{1}^{*}\left(\varphi(a)-\varphi\left(s_{0}\right)\right)+\tilde{k}_{2}^{*}\left(\varphi(a)-\varphi\left(s_{0}\right)\right)^{2}+\cdots+\tilde{k}_{n-1}^{*}\left(\varphi(a)-\varphi\left(s_{0}\right)\right)^{n-1},
\end{aligned}
$$

where $n-1<\sigma^{*}<n$ and $\tilde{k}_{0}^{*}, \tilde{k}_{1}^{*}, \ldots, \tilde{k}_{n-1}^{*} \in \mathbb{R}[15]$.

Regarding $(N,\|\cdot\|)$ a normed space, the classes $\mathbb{P}_{\mathbb{C L}}(N)$ (closed), $\mathbb{P}_{\mathbb{B N}}(N)$ (bounded), $\mathbb{P}_{\mathbb{C P}}(N)$ (compact), and $\mathbb{P}_{\mathbb{C V}}(N)$ (convex) consist of a respective form of the subsets of $N$.

Definition 1 ([30]) Consider $v: \mathbb{R} \rightarrow \mathbb{R}$ as a real-valued function and $v$ as a multifunction. 
(i) $F$ is u.s.c on $N$ if $F\left(v^{*}\right) \in \mathbb{P}_{\mathbb{C L}}(N)$ for any $v^{*} \in N$ and also a neighborhood $N_{0}^{*}$ of $v^{*}$ exists subject to $F\left(N_{0}^{*}\right) \subseteq \mathbb{V}$ for $\mathbb{V} \subseteq N$ in which $\mathbb{V}$ is an arbitrary open set.

(ii) A real-valued map $v: \mathbb{R} \rightarrow \mathbb{R}$ is upper semi-continuous such that $\limsup _{n \rightarrow \infty} v\left(\varsigma_{n}\right) \leq v(\varsigma)$ for each $\left\{\zeta_{n}\right\}_{n \geq 1}$ with $\varsigma_{n} \rightarrow \varsigma$.

Definition 2 ([30]) A metric attributed to Pompeiu-Hausdorff $\mathbb{H}_{d}:(\mathbb{P}(N))^{2} \rightarrow \mathbb{R} \cup\{\infty\}$ is defined as

$$
\mathbb{H}_{d}\left(Q_{1}^{*}, Q_{2}^{*}\right)=\max \left\{\sup _{q_{1}^{*} \in Q_{1}^{*}} d\left(q_{1}^{*}, Q_{2}^{*}\right), \sup _{q_{2}^{*} \in Q_{2}^{*}} d\left(Q_{1}^{*}, q_{2}^{*}\right)\right\}
$$

where $d$ is considered to be the metric of $N$ and also $d\left(Q_{1}^{*}, q_{2}^{*}\right)=\inf _{q_{1}^{*} \in Q_{1}^{*}} d\left(q_{1}^{*}, q_{2}^{*}\right)$ and $d\left(q_{1}^{*}, Q_{2}^{*}\right)=\inf _{q_{2}^{*} \in Q_{2}^{*}} d\left(q_{1}^{*}, q_{2}^{*}\right)$.

Definition 3 ([30]) For $F: N \rightarrow \mathbb{P}_{\mathbb{C L}}(N)$ and $v_{1}, v_{2} \in N$, let the following inequality hold:

$$
\mathbb{H}_{d}\left(F\left(v_{1}\right), F\left(v_{2}\right)\right) \leq \ell d\left(v_{1}, v_{2}\right)
$$

Then $F$ is said to be: (H1) a Lipschitz map if $\ell>0$; (H2) a contraction if $0<\ell<1$.

Definition 4 ([30]) An element $\mu^{*} \in N$ is termed an endpoint of a multi-valued function $L: N \rightarrow \mathbb{P}(N)$ whenever we get $L\left(\mu^{*}\right)=\left\{\mu^{*}\right\}$. The multi-valued map $\Phi$ has an approximate endpoint criterion (AEP) if

$$
\inf _{\mu_{1}^{*} \in N} \sup _{\mu_{2}^{*} \in L\left(\mu_{1}^{*}\right)} \mathrm{d}\left(\mu_{1}^{*}, \mu_{2}^{*}\right)=0
$$

\section{Definition 5 ([30])}

(i) $\Phi: \mathbb{S} \times \mathbb{R} \rightarrow \mathbb{P}(\mathbb{R})$ is Caratheodory if $\varsigma \mapsto \Phi(\varsigma, u)$ is measurable for any $u \in \mathbb{R}$ and $u \mapsto \Phi(\varsigma, u)$ is u.s.c for a.e. $\varsigma \in \mathbb{S}$.

(ii) A Caratheodory multifunction $\Phi: \mathbb{S} \times \mathbb{R} \rightarrow \mathbb{P}(\mathbb{R})$ is $L^{1}$-Caratheodory if, for any $\eta>0, \mu_{\eta} \in L^{1}\left(\mathbb{S}, \mathbb{R}_{+}\right)$exists subject to $\|\Phi(\varsigma, u)\|=\sup _{\varsigma \in \mathbb{S}}\{|v|: v \in \Phi(\varsigma, u)\} \leq \mu_{\eta}(\varsigma)$ for all $|u| \leq \eta$ and for almost all $\varsigma \in \mathbb{S}$.

We will use a particular set of functions and properties that are relevant to the argumentative purposes of this article. This mapping category was first defined by Samet, Vetro, and Vetro [25] in 2012. Regard $\Psi$ consisting of all nondecreasing functions $\psi:[0, \infty) \rightarrow$ $[0, \infty)$ such that, for every $a>0, \sum_{n=1}^{\infty} \psi^{n}(a)<\infty$ and and $\alpha: N^{2} \rightarrow \mathbb{R}_{\geq 0}$. [25] The selfmap $T$ on $(N, \mathrm{~d})$ is regarded as $\alpha$-admissible if $\alpha\left(T \mu_{1}^{*}, T \mu_{2}^{*}\right) \geq 1$ whenever $\alpha\left(\mu_{1}^{*}, \mu_{2}^{*}\right) \geq 1$ and is $\alpha-\psi$-contraction

$$
\alpha\left(\mu_{1}^{*}, \mu_{2}^{*}\right) \mathrm{d}\left(T \mu_{1}^{*}, T \mu_{2}^{*}\right) \leq \psi\left(\mathrm{d}\left(\mu_{1}^{*}, \mu_{2}^{*}\right)\right)
$$

for every $\mu_{1}^{*}, \mu_{2}^{*} \in N$. Moreover, the normed space $N$ admits that $\left(\mathbf{P}_{1}\right)$ if $\left\{\mu_{n}^{*}\right\}_{n \geq 1}$ is any sequence in $N$ with $\alpha\left(\mu_{n}^{*}, \mu_{n+1}^{*}\right) \geq 1$ and $\mu_{n}^{*}$ tends to $\mu^{*}$, for every $n \geq 1$, the inequality $\alpha\left(\mu_{n}^{*}, \mu^{*}\right) \geq 1$ follows. A generalized version of $\alpha-\psi$-contraction was given by [31, 32]. Karapinar et al. [33] proved some new fixed point results concerning $\alpha-\psi$-contraction and applied them for the existence of solution of FDEs. 
After the notions presented by Samet et al. [25], next year, Mohammadi et al. came up with another classification of such maps generalizing the previous one [34]. The set-valued map $L$ having bounded and closed values on $N$ is regarded as $\alpha$-admissible if, for every $\mu_{1}^{*} \in N$ and $\mu_{2}^{*} \in L \mu_{1}^{*}, \alpha\left(\mu_{2}^{*}, \mu_{3}^{*}\right) \geq 1$ whenever $\alpha\left(\mu_{1}^{*}, \mu_{2}^{*}\right) \geq 1$ for every $\mu_{3}^{*} \in L \mu_{2}^{*}$ and is $\alpha-\psi$-contractive if

$$
\alpha\left(\mu_{1}^{*}, \mu_{2}^{*}\right) \mathbb{H}_{\mathrm{d}}\left(L \mu_{1}^{*}, L \mu_{2}^{*}\right) \leq \psi\left(\mathrm{d}\left(\mu_{1}^{*}, \mu_{2}^{*}\right)\right)
$$

for every member $\mu_{1}^{*}, \mu_{2}^{*} \in N$. Moreover, the normed space $N$ admits that $\left(\mathbf{P}_{2}\right)$ if $\left\{\mu_{n}^{*}\right\}_{n \geq 1}$ is any sequence in $N$ with $\alpha\left(\mu_{n}^{*}, \mu_{n+1}^{*}\right) \geq 1$ and $\mu_{n}^{*}$ tends to $\mu^{*}$, there is a sequence $\left\{\mu_{n}^{*}\right\}$ containing a subsequence $\left\{\mu_{n_{k}}^{*}\right\}$ satisfying $\alpha\left(\mu_{n_{k}}^{*}, \mu^{*}\right) \geq 1$ for all natural numbers $k$.

Our claims are based on the following theorems until the conclusion of this study.

Theorem 1 ([25]) Regard ( $N, \mathrm{~d})$, a complete metric space, $\psi \in \Psi$, a real-valued map $\alpha$ on $N^{2}$. Regarding a self-map T on $N$ as $\alpha$-admissible and $\alpha-\psi$-contractive with $\alpha\left(\mu_{0}^{*}, T \mu_{0}^{*}\right) \geq$ 1 for some $\mu_{0}^{*} \in N$ together with $N$ admitting property $\left(\mathbf{P}_{1}\right)$. This yields that a fixed point of the map $T$ exists.

Theorem 2 ([35]) Regarding $N$ a complete normed space, operators $T_{1}$ and $T_{2}$ defined on a bounded, convex and closed subset $\mathbb{B} \neq \emptyset$ of $N$ confirms that, for every $\mu_{1}^{*}, \mu_{2}^{*} \in \mathbb{B}$, $T_{1} \mu_{1}^{*}+T_{2} \mu_{2}^{*} \in \mathbb{B}$, operator $T_{1}$ is continuous and compact while that of $T_{2}$ is contractive. Then there is a member $\mu^{*}$ of $\mathbb{B}$ with $T_{1} \mu_{1}^{*}+T_{2} \mu_{2}^{*}=\mu^{*}$.

Theorem 3 ([34]) Regard $(N, \mathrm{~d})$ a complete metric space, $\psi \in \Psi$, a real-valued map $\alpha$ on $N^{2}$. Regard a bounded and closed set-valued map $L$ on $N$ as $\alpha$-admissible and $\alpha-\psi$ contractive with $\alpha\left(\mu_{0}^{*}, \mu_{1}^{*}\right) \geq 1$ for some $\mu_{0}^{*} \in N$ and $\mu_{1}^{*} \in L \mu_{0}^{*}$ together with $N$ admitting $\left(\mathbf{P}_{2}\right)$. This yields that L possesses a fixed point.

Theorem 4 ([30]) Regard ( $N$, d) a complete metric space, $\psi \in \Psi$ is upper semi-continuous together with $\liminf _{a \rightarrow \infty}(a-\psi(a))>0$ for $a>0$, a bounded and closed set-valued map $L$ on $N$ admits the inequality $\mathbb{H}_{\mathrm{d}}\left(L \mu_{1}^{*}, L \mu_{2}^{*}\right) \leq \psi\left(\mathrm{d}\left(\mu_{1}^{*}, \mu_{2}^{*}\right)\right)$ for every $\mu_{1}^{*}, \mu_{2}^{*} \in N$. This yields that the map L follows the approximate endpoint property iff there exists a unique endpoint of it.

\section{Fractional $\varphi$-BVP (1)}

In the current situation of study we are beginning to pursue the basic deductions of a potential solution of problem (1) via nonlinear methods in the theory of fixed point of an operator under consideration. In respect of achieving the goals, regard $N=\left\{\mu^{*}(a)\right.$ : $\left.\mu^{*}(a) \in C(J, \mathbb{R})\right\}$ a Banach space supplied with $\left\|\mu^{*}\right\|_{N}=\sup _{a \in J}\left|\mu^{*}(a)\right|$ for all $\mu^{*} \in N$. The solution of the suggested problem (1) is presented in the next lemma in the form of an integral equation which helps us to present the existence criteria.

Lemma 3.1 Let $\Phi^{*} \in C(J, \mathbb{R})$ be the continuous function, then the function $\mu^{*}(a) \in N$ is a solution for the boundary value problem

$$
\left\{\begin{array}{l}
{ }^{C} D_{0}^{\sigma^{*}, \varphi} \mu^{*}(a)=\Phi^{*}(a), \quad a \in J \\
\mu^{*}\left(s_{0}\right)=0 \\
\mu^{*}(M)=\sum_{i=1}^{m} \beta_{i}{ }^{R L} I_{0}^{p_{i}, \varphi} \mu^{*}\left(\xi_{i}\right),
\end{array}\right.
$$


if and only if $\mu^{*}(a)$ is a solution for the following:

$$
\mu^{*}(a)={ }^{R L} I_{0}^{\sigma^{*}, \varphi} \Phi^{*}(a)-\frac{\left(\varphi(a)-\varphi\left(s_{0}\right)\right)}{\Lambda}\left[{ }^{R L} I_{0}^{\sigma^{*}, \varphi} \Phi^{*}(M)-\sum_{i=1}^{m} \beta_{i}{ }^{R L} I_{0}^{p_{i}+\sigma^{*}, \varphi} \Phi^{*}\left(\xi_{i}\right)\right] .
$$

Proof Let $\mu^{*}(a)$ be the solution of BVP. Choose $k_{0}, k_{1} \in \mathbb{R}$ such that

$$
\mu^{*}(a)={ }^{R L} I_{0}^{\sigma^{*}, \varphi} \Phi^{*}(a)-k_{0}-k_{1}\left(\varphi(a)-\varphi\left(s_{0}\right)\right) .
$$

By using $\mu\left(s_{0}\right)=0$, we have $k_{0}=0$

$$
\mu^{*}(a)={ }^{R L} I_{0}^{\sigma^{*}, \varphi} \Phi^{*}(a)-k_{1}\left(\varphi(a)-\varphi\left(s_{0}\right)\right) .
$$

Operating fractional Riemann-Liouville integral having order $p_{i}>0$ for (11), we have

$$
\begin{aligned}
& { }^{R L} I_{0}^{p_{i}, \varphi} \mu^{*}(a)={ }^{R L} I_{0}^{p_{i}+\sigma^{*}, \varphi} \Phi^{*}(a)-k_{1} \frac{\left(\varphi(a)-\varphi\left(s_{0}\right)\right)^{p_{i}+1}}{\Gamma\left(p_{i}+2\right)} \\
& { }^{R L} I_{0}^{\sigma^{*}, \varphi} \Phi^{*}(M)-k_{1}\left(\varphi(M)-\varphi\left(s_{0}\right)\right)=\sum_{i=1}^{m} \beta_{i}{ }^{R L} I_{0}^{p_{i}+\sigma^{*}, \varphi} \Phi^{*}\left(\xi_{i}\right)-k_{1} \sum_{i=1}^{m} \beta_{i} \frac{\xi^{p_{i}+1}}{\Gamma\left(p_{i}+2\right)} .
\end{aligned}
$$

Thus

$$
k_{1}\left[\left(\varphi(M)-\varphi\left(s_{0}\right)\right)-\sum_{i=1}^{m} \beta_{i} \frac{\xi^{p_{i}+1}}{\Gamma\left(p_{i}+2\right)}\right]={ }^{R L} I_{0}^{\sigma^{*}, \varphi} \Phi^{*}(M)-\sum_{i=1}^{m} \beta_{i}{ }^{R L} I_{0}^{p_{i}+\sigma^{*}, \varphi} \Phi^{*}\left(\xi_{i}\right)
$$

implies that

$$
\begin{aligned}
& \Lambda=\left(\varphi(M)-\varphi\left(s_{0}\right)\right)-\sum_{i=1}^{m} \beta_{i} \frac{\xi^{p_{i}+1}}{\Gamma\left(p_{i}+2\right)} \neq 0, \\
& k_{1}=\frac{1}{\Lambda}\left[{ }^{R L} I_{0}^{\sigma^{*}, \varphi} \Phi^{*}(M)-\sum_{i=1}^{n} \beta_{i}{ }^{R L} I_{0}^{p_{i}+\sigma^{*}, \varphi} \Phi^{*}\left(\xi_{i}\right)\right], \\
& \mu^{*}(a)={ }^{R L} I_{0}^{\sigma^{*}, \varphi} \Phi^{*}(a)-\frac{\left(\varphi(a)-\varphi\left(s_{0}\right)\right)}{\Lambda}\left[{ }^{R L} I_{0}^{\sigma^{*}, \varphi} \Phi^{*}(M)-\sum_{i=1}^{m} \beta_{i}{ }^{R L} I_{0}^{p^{p}+\sigma^{*}, \varphi} \Phi^{*}\left(\xi_{i}\right)\right],
\end{aligned}
$$

where $\Lambda$ is defined in (15).

Throughout this work we use the following:

$$
\begin{aligned}
& { }^{R L} I_{0}^{\sigma^{*}, \varphi} \hbar\left(a, \mu^{*}(a)\right)=\frac{1}{\Gamma\left(\sigma^{*}\right)} \int_{s_{0}}^{a} \varphi^{\prime}(q)(\varphi(a)-\varphi(q))^{\sigma^{*}-1} \hbar\left(q, \mu^{*}(q)\right) \mathrm{d} q \\
& { }^{R L} I_{0}^{p_{i}+\sigma^{*}, \varphi} \hbar\left(\xi_{i}, \mu^{*}\left(\xi_{i}\right)\right)=\frac{1}{\Gamma\left(p_{i}+\sigma^{*}\right)} \int_{s_{0}}^{\xi_{i}} \varphi^{\prime}(q)\left(\varphi\left(\xi_{i}\right)-\varphi(q)\right)^{p_{i}+\sigma^{*}-1} \hbar\left(q, \mu^{*}(q)\right) \mathrm{d} q,
\end{aligned}
$$

where $\xi_{i} \in J, i=1,2, \ldots, m$. Let $N=C(J, \mathbb{R})$. 
We define the operator $T: N \rightarrow N$ by

$$
\begin{aligned}
T \mu^{*}(a)= & { }^{R L} I_{0}^{\sigma^{*}, \varphi} \hbar\left(a, \mu^{*}(a)\right)-\frac{\left(\varphi(a)-\varphi\left(s_{0}\right)\right)}{\Lambda}\left[{ }^{R L} I_{0}^{\sigma^{*}, \varphi} \hbar\left(M, \mu^{*}(M)\right)\right. \\
& \left.-\sum_{i=1}^{m} \beta_{i}{ }^{R L} I_{0}^{p_{i}+\sigma^{*}, \varphi} \hbar\left(\xi_{i}, \mu^{*}\left(\xi_{i}\right)\right)\right]
\end{aligned}
$$

It is noted that BVP has a solution if $T$ has a fixed point.

For the sake of convenience in writing, we set

$$
\begin{aligned}
\Omega_{1}= & \frac{\left(\left|\varphi(M)-\varphi\left(s_{0}\right)\right|\right)^{\sigma^{*}}}{\Gamma\left(\sigma^{*}+1\right)}+\frac{\left(\left|\left(\varphi(M)-\varphi\left(s_{0}\right)\right)\right|\right)^{\sigma^{*}+1}}{|\Lambda| \Gamma\left(\sigma^{*}+1\right)} \\
& +\sum_{i=1}^{m} \beta_{i} \frac{m\left(\left|\left(\varphi(M)-\varphi\left(s_{0}\right)\right)\right|\right)^{p_{i}+\sigma^{*}+1}}{|\Lambda| \Gamma\left(p_{i}+\sigma^{*}+1\right)}, \\
\Omega_{2}= & \frac{\left\|\eta^{*}\right\|\left(\left|\varphi(M)-\varphi\left(s_{0}\right)\right|\right)^{\sigma^{*}+1}}{|\Lambda| \Gamma\left(\sigma^{*}+1\right)}+\sum_{i=1}^{m} m \beta_{i} \frac{\left\|\eta^{*}\right\|\left(\left|\varphi(M)-\varphi\left(s_{0}\right)\right|\right)^{p_{i} \sigma^{*}+1}}{|\Lambda| \Gamma\left(p_{i}+\sigma^{*}+1\right)} .
\end{aligned}
$$

Theorem 5 Let $\psi \in \Psi, G: \mathbb{R}^{2} \rightarrow \mathbb{R}$ be a mapping, $\hbar: J \times N \rightarrow N$ be a continuous function, suppose that

$C_{1}:\left|\hbar\left(a, \mu_{1}^{*}(a)\right)-\hbar\left(a, \mu_{2}^{*}(a)\right)\right| \leq \lambda^{*} \psi\left(\left|\mu_{1}^{*}-\mu_{2}^{*}\right|\right)$ for all $\mu_{1}^{*}, \mu_{2}^{*} \in N$ and

$$
G\left(\left(\mu_{1}^{*}, \mu_{2}^{*}\right)\right) \geq 0
$$

$C_{2}$ : There exists $\mu_{0}^{*} \in N$ such that

$$
G\left(\left(\mu_{0}^{*}(a), T \mu_{0}^{*}(a)\right) \geq 0\right.
$$

for all $a \in J$, and

$$
G\left(\left(\mu_{1}^{*}(a), \mu_{2}^{*}(a)\right)\right) \geq 0
$$

implies

$$
G\left(\left(T \mu_{1}^{*}(a), T \mu_{2}^{*}(a)\right)\right) \geq 0
$$

for every $\mu_{1}^{*}, \mu_{2}^{*} \in N$ and $a \in J$.

$C_{3}$ : For every sequence $\left\{\mu_{n}^{*}\right\}_{n \geq 1}$ in $N$ with $\mu_{n}^{*} \rightarrow \mu^{*}$ and

$$
G\left(\mu_{n}^{*}(a), \mu_{n+1}^{*}(a)\right) \geq 0
$$

for every $n \in \mathbb{N}$ and $a \in J$, we get

$$
G\left(\left(\mu_{n}^{*}(a), \mu^{*}(a)\right)\right) \geq 0 .
$$

Then at least one solution of problem (1) exists. 
Proof Assume $\mu_{1}^{*}, \mu_{2}^{*} \in N$ such that $G\left(\left(\mu_{1}^{*}(a), \mu_{2}^{*}(a)\right)\right) \geq 0$ for all $a \in J$. Then

$$
\begin{aligned}
\left|T \mu_{1}^{*}(a)-T \mu_{2}^{*}(a)\right| & \\
\leq & \frac{1}{\Gamma\left(\sigma^{*}\right)} \int_{s_{0}}^{a} \varphi^{\prime}(q)(\varphi(a)-\varphi(q))^{\sigma^{*}-1}\left|\hbar\left(q, \mu_{1}^{*}(q)\right)-\hbar\left(q, \mu_{2}^{*}(q)\right)\right| \mathrm{d} q \\
& +\frac{\left|\left(\varphi(a)-\varphi\left(s_{0}\right)\right)\right|}{|\Lambda| \Gamma\left(\sigma^{*}\right)} \int_{s_{0}}^{M} \varphi^{\prime}(q)(\varphi(M)-\varphi(q))^{\sigma^{*}-1}\left|\hbar\left(q, \mu_{1}^{*}(q)\right)-\hbar\left(q, \mu_{2}^{*}(q)\right)\right| \mathrm{d} q \\
& +\sum_{i=1}^{m} \beta_{i} \frac{\left|\left(\varphi(a)-\varphi\left(s_{0}\right)\right)\right|}{|\Lambda| \Gamma\left(p_{i}+\sigma^{*}\right)} \\
& \times \int_{s_{0}}^{\xi_{i}} \varphi^{\prime}(q)\left(\varphi\left(\xi_{i}\right)-\varphi(q)\right)^{p_{i}+\sigma^{*}-1}\left|\hbar\left(q, \mu_{1}^{*}(q)\right)-\hbar\left(q, \mu_{2}^{*}(q)\right)\right| \mathrm{d} q \\
\leq & \frac{\lambda^{*}}{\Gamma\left(\sigma^{*}\right)} \int_{s_{0}}^{a} \varphi^{\prime}(q)(\varphi(a)-\varphi(q))^{\sigma^{*}-1} \psi\left(\left|\mu_{1}^{*}-\mu_{2}^{*}\right|\right) \mathrm{d} q \\
& +\frac{\lambda^{*}\left|\left(\varphi(a)-\varphi\left(s_{0}\right)\right)\right|}{|\Lambda| \Gamma\left(\sigma^{*}\right)} \int_{s_{0}}^{M} \varphi^{\prime}(q)(\varphi(M)-\varphi(q))^{\sigma^{*}-1} \psi\left(\left|\mu_{1}^{*}-\mu_{2}^{*}\right|\right) \mathrm{d} q \\
& +\sum_{i=1}^{m} \beta_{i} \frac{\lambda^{*}\left|\left(\varphi(a)-\varphi\left(s_{0}\right)\right)\right|}{|\Lambda| \Gamma\left(p_{i}+\sigma^{*}\right)} \int_{s_{0}}^{\xi_{i}} \varphi^{\prime}(q)\left(\varphi\left(\xi_{i}\right)-\varphi(q)\right)^{p_{i}+\sigma^{*}-1} \psi\left(\left|\mu_{1}^{*}-\mu_{2}^{*}\right|\right) \mathrm{d} q \\
\leq & \frac{\lambda^{*} \psi\left(\left\|\mu_{1}^{*}-\mu_{2}^{*}\right\|\right)\left(\left|\varphi(a)-\varphi\left(s_{0}\right)\right|\right)^{\sigma^{*}}}{\Gamma\left(\sigma^{*}+1\right)}+\frac{\lambda^{*} \psi\left(\left\|\mu_{1}^{*}-\mu_{2}^{*}\right\|\right)\left(\left|\left(\varphi(a)-\varphi\left(s_{0}\right)\right)\right|\right)^{\sigma^{*}+1}}{|\Lambda| \Gamma\left(\sigma^{*}+1\right)} \\
& +\sum_{i=1}^{m} \beta_{i} \frac{\lambda^{*} \psi\left(\left\|\mu_{1}^{*}-\mu_{2}^{*}\right\|\right)(|(\varphi(a)-\varphi(q))|)^{p_{i}+\sigma^{*}+1}}{|\Lambda| \Gamma\left(p_{i}+\sigma^{*}+1\right)} \\
= & \lambda^{*}\left\{\frac{\left(\left|\varphi(M)-\varphi\left(s_{0}\right)\right|\right)^{\sigma^{*}}}{\Gamma\left(\sigma^{*}+1\right)}+\frac{\left(\left|\left(\varphi(M)-\varphi\left(s_{0}\right)\right)\right|\right)^{\sigma^{*}+1}}{|\Lambda| \Gamma\left(\sigma^{*}+1\right)}\right. \\
& \left.+\sum_{i=1}^{m} \beta_{i} \frac{m\left(\left|\left(\varphi(M)-\varphi\left(s_{0}\right)\right)\right|\right)^{p_{i}+\sigma^{*}+1}}{|\Lambda| \Gamma\left(p_{i}+\sigma^{*}+1\right)}\right\} \psi\left(\left\|\mu_{1}^{*}-\mu_{2}^{*}\right\|\right) . \\
&
\end{aligned}
$$

Hence, $\left|T \mu_{1}^{*}(a)-T \mu_{2}^{*}(a)\right| \leq \Omega_{1} \lambda^{*} \psi\left(\left\|\mu_{1}^{*}-\mu_{2}^{*}\right\|\right)$. We regard a nonnegative function $\alpha$ on $N \times N$ by

$$
\alpha\left(\mu_{1}^{*}, \mu_{2}^{*}\right)= \begin{cases}1 & \text { if } G\left(\left(\mu_{1}^{*}(a), \mu_{2}^{*}(a)\right)\right) \geq 0, \\ 0 & \text { otherwise }\end{cases}
$$

for all $\mu_{1}^{*}, \mu_{2}^{*} \in N$. Then we have $\alpha\left(\mu_{1}^{*}, \mu_{2}^{*}\right) \mathrm{d}\left(T \mu_{1}^{*}, T \mu_{2}^{*}\right) \leq \psi\left(\mathrm{d}\left(\mu_{1}^{*}, \mu_{2}^{*}\right)\right)$ for all $\mu_{1}^{*}, \mu_{2}^{*} \in N$, which indicates that the operator $T$ is an $\alpha-\psi$-contractive type. In addition, one can easily verify that $T$ is $\alpha$-admissible and $1 \leq \alpha\left(\mu_{0}^{*}, T \mu_{0}^{*}\right)$. Further, assume that a sequence $\left\{\mu_{n}^{*}\right\}$ in $N$ tends to $\mu^{*}$ with $1 \leq \alpha\left(\mu_{n}^{*}, \mu_{n+1}^{*}\right)$ for all $n$. Due to the structure of $\alpha$, it implies that

$$
G\left(\left(\mu_{n}^{*}(a), \mu_{n+1}^{*}(a)\right)\right) \geq 0 .
$$

Due to assertion $\left(C_{3}\right)$, we have

$$
G\left(\left(\mu_{n}^{*}(a), \mu^{*}(a)\right)\right) \geq 0,
$$


which asserts that $\alpha\left(\mu_{n}^{*}, \mu^{*}\right) \geq 1$ for all $n$. Therefore $N$ admits property $\left(\mathbf{P}_{1}\right)$. Theorem 1 is thus applied, and we find that the operator $T$ possesses $\mu^{*} \in N$ as a fixed point, which in turn is a solution of the proposed BVP (1). The proof is complete.

Theorem 6 Regard a continuous function $\hbar: J \times N \rightarrow N$. We assume the following:

$C_{4}$ : A continuous function $L^{*}$ on $J$ exists such that

$$
\left|\hbar\left(a, \mu_{1}^{*}\right)-\hbar\left(a, \mu_{2}^{*}\right)\right| \leq L^{*}(a)\left(\left|\mu_{1}^{*}-\mu_{2}^{*}\right|\right)
$$

for all $a \in J$ and $\mu_{1}^{*}, \mu_{2}^{*} \in N$.

$C_{5}$ : There exist a continuous function $\eta^{*}: J \rightarrow \mathbb{R}^{+}$and a nondecreasing function $\psi: J \rightarrow$ $\mathbb{R}^{+}$such that

$$
\hbar\left(a, \mu_{1}^{*}\right) \leq \eta^{*}(a) \psi\left(\left|\mu_{1}^{*}\right|\right)
$$

for all $a \in J$ and $\mu_{1}^{*} \in N$. Then the fractional BVP has at least one solution whenever $K^{*}=\left\|L^{*}\right\| \Omega_{1}$ where $\left\|L^{*}\right\|=\sup _{a \in J}\left|L^{*}(a)\right|$.

Proof Let $\left\|\eta^{*}\right\|=\sup _{a \in J}\left|\eta^{*}(a)\right|$ and $\epsilon \geq\left\|m^{*}\right\|\left\|\eta^{*}\right\| \Omega_{1}$ Let us define a set $V_{\epsilon}=\left\{\mu^{*} \in N\right.$ : $\left.\left\|\mu^{*}\right\| \leq \epsilon\right\}$. It is easy to verify that $V_{\epsilon}$ is a nonempty, convex, closed, bounded subset of Banach space $N$. We now define two operators $T^{1}$ and $T^{2}$ on a set $V_{\epsilon}$ by

$$
\begin{aligned}
T^{1} \mu^{*}(a)= & \frac{1}{\Gamma\left(\sigma^{*}\right)} \int_{s_{0}}^{a} \varphi^{\prime}(q)(\varphi(a)-\varphi(q))^{\sigma^{*}-1} \hbar\left(q, \mu^{*}(q)\right) \mathrm{d} q \\
T^{2} \mu^{*}(a)= & \frac{\varphi(a)-\varphi\left(s_{0}\right)}{\Lambda}\left[\frac{1}{\Gamma\left(\sigma^{*}\right)} \int_{s_{0}}^{M} \varphi^{\prime}(q)(\varphi(M)-\varphi(q))^{\sigma^{*}-1} \hbar\left(q, \mu^{*}(q)\right) \mathrm{d} q\right. \\
& \left.+\sum_{i=1}^{m} \beta_{i} \frac{1}{\Gamma\left(p_{i}+\sigma^{*}\right)} \int_{s_{0}}^{\xi_{i}} \varphi^{\prime}(q)\left(\varphi\left(\xi_{i}\right)-\varphi(q)\right)^{p_{i}+\sigma^{*}-1} \hbar\left(q, \mu^{*}(q)\right) \mathrm{d} q\right] .
\end{aligned}
$$

For all $a \in J$, put $\left\|m^{*}\right\|=\sup _{a \in J} \psi\left(\left\|\mu^{*}(a)\right\|\right)$, for $\mu_{1}^{*}, \mu_{2}^{*} \in V_{\epsilon}$ we have

$$
\begin{aligned}
\left|T^{1} \mu_{1}^{*}(a)+T^{2} \mu_{2}^{*}(a)\right| & \frac{1}{\Gamma\left(\sigma^{*}\right)} \int_{s_{0}}^{a} \varphi^{\prime}(q)(\varphi(a)-\varphi(q))^{\sigma^{*}-1}\left|\hbar\left(q, \mu_{1}^{*}(q)\right)\right| \mathrm{d} q \\
& +\frac{\varphi(a)-\varphi\left(s_{0}\right)}{|\Lambda|}\left[\frac{1}{\Gamma\left(\sigma^{*}\right)} \int_{s_{0}}^{M} \varphi^{\prime}(q)(\varphi(M)-\varphi(q))^{\sigma^{*}-1}\left|\hbar\left(q, \mu_{2}^{*}(q)\right)\right| \mathrm{d} q\right. \\
& \left.+\sum_{i=1}^{m} \beta_{i} \frac{1}{\Gamma\left(p_{i}+\sigma^{*}\right)} \int_{s_{0}}^{\xi_{i}} \varphi^{\prime}(q)\left(\varphi\left(\xi_{i}\right)-\varphi(q)\right)^{p_{i}+\sigma^{*}-1}\left|\hbar\left(q, \mu_{2}^{*}(q)\right)\right| \mathrm{d} q\right] \\
\leq & \frac{1}{\Gamma\left(\sigma^{*}\right)} \int_{s_{0}}^{a} \varphi^{\prime}(q)(\varphi(a)-\varphi(q))^{\sigma^{*}-1} \eta^{*}(q) \psi\left(\left|\mu_{1}^{*}(q)\right|\right) \mathrm{d} q \\
& +\frac{\varphi(a)-\varphi\left(s_{0}\right)}{|\Lambda|}\left[\frac{1}{\Gamma\left(\sigma^{*}\right)} \int_{s_{0}}^{M} \varphi^{\prime}(q)(\varphi(M)-\varphi(q))^{\sigma^{*}-1} \eta^{*}(q) \psi\left(\left|\mu_{2}^{*}(q)\right|\right) \mathrm{d} q\right. \\
& \left.+\sum_{i=1}^{m} \beta_{i} \frac{1}{\Gamma\left(p_{i}+\sigma^{*}\right)} \int_{s_{0}}^{\xi_{i}} \varphi^{\prime}(q)\left(\varphi\left(\xi_{i}\right)-\varphi(q)\right)^{p_{i}+\sigma^{*}-1} \eta^{*}(q) \psi\left(\left|\mu_{2}^{*}(q)\right|\right) \mathrm{d} q\right]
\end{aligned}
$$




$$
\begin{aligned}
\leq & \frac{\left\|\eta^{*}\right\| \varphi(M)-\left.\varphi\left(s_{0}\right)\right|^{\sigma^{*}}}{\Gamma\left(\sigma^{*}+1\right)} \psi\left(\left\|\mu^{*}\right\|\right)+\frac{\left\|\eta^{*}\right\|\left|\varphi(M)-\varphi\left(s_{0}\right)\right|^{\sigma^{*}+1}}{|\Lambda| \Gamma\left(\sigma^{*}+1\right)} \psi\left(\left\|\mu^{*}\right\|\right) \\
& +m \sum_{i=1}^{m} \beta_{i} \frac{\left\|\eta^{*}\right\|\left|\varphi(M)-\varphi\left(s_{0}\right)\right|^{p_{i}+\sigma^{*}+1}}{|\Lambda| \Gamma\left(p_{i}+\sigma^{*}+1\right)} \psi\left(\left\|\mu^{*}\right\|\right) \\
= & \left\|\eta^{*}\right\| \Omega_{1}\left\|m^{*}\right\| \leq \epsilon .
\end{aligned}
$$

Hence $\left|T^{1} \mu_{1}^{*}(a)+T^{2} \mu_{2}^{*}(a)\right| \leq \epsilon$, which implies that $\left|T^{1} \mu_{1}^{*}(a)+T^{2} \mu_{2}^{*}(a)\right| \in V_{\epsilon}$. By hypothesis, the function $\hbar$ is continuous, which thus implies that the operator $T^{1}$ is continuous. Moreover,

$$
\begin{aligned}
\left|T^{1} \mu^{*}(a)\right| & \leq \frac{1}{\Gamma\left(\sigma^{*}\right)} \int_{s_{0}}^{a} \varphi^{\prime}(q)(\varphi(a)-\varphi(q))^{\sigma^{*}-1}\left|\hbar\left(q, \mu^{*}(q)\right)\right| \mathrm{d} q \\
& \leq \frac{1}{\Gamma\left(\sigma^{*}\right)} \int_{s_{0}}^{a} \varphi^{\prime}(q)(\varphi(a)-\varphi(q))^{\sigma^{*}-1} \eta^{*}(q) \psi\left(\left\|\mu^{*}\right\|\right) \mathrm{d} q \\
& \leq \frac{\left\|\eta^{*}\right\|\left\|m^{*}\right\|\left|\varphi(M)-\varphi\left(s_{0}\right)\right|^{\sigma^{*}}}{\Gamma\left(\sigma^{*}+1\right)}
\end{aligned}
$$

for all $\mu^{*} \in V_{\epsilon}$. Hence,

$$
\left\|T^{1} \mu^{*}(a)\right\| \leq \frac{\left\|m^{*}\right\|\left\|\eta^{*}\right\|\left|\varphi(M)-\varphi\left(s_{0}\right)\right|^{\sigma^{*}}}{\Gamma\left(\sigma^{*}+1\right)}
$$

which implies the uniform boundedness of the operator $T^{1}$ on $V_{\epsilon}$. Over the next move, we would pursue that $T^{1}$ as a compact operator on $V_{\epsilon}$. To achieve this, we take $a_{1}, a_{2} \in J$ with $a_{2}>a_{1}$, thus we have

$$
\begin{aligned}
&\left|T^{1} \mu^{*}\left(a_{2}\right)-T^{1} \mu^{*}\left(a_{1}\right)\right| \\
&=\frac{1}{\Gamma\left(\sigma^{*}\right)} \int_{s_{0}}^{a_{2}} \varphi^{\prime}(q)\left(\varphi\left(a_{2}\right)-\varphi(q)\right)^{\sigma^{*}-1}\left|\hbar\left(q, \mu^{*}(q)\right)\right| \mathrm{d} q \\
&-\frac{1}{\Gamma\left(\sigma^{*}\right)} \int_{s_{0}}^{a_{1}} \varphi^{\prime}(q)\left(\varphi\left(a_{1}\right)-\varphi(q)\right)^{\sigma^{*}-1}\left|\hbar\left(q, \mu^{*}(q)\right)\right| \mathrm{d} q \\
&= \frac{1}{\Gamma\left(\sigma^{*}\right)} \int_{s_{0}}^{a_{1}} \varphi^{\prime}(q)\left[\left(\varphi\left(a_{2}\right)-\varphi(q)\right)^{\sigma^{*}-1}-\left(\varphi\left(a_{1}\right)-\varphi(q)\right)^{\sigma^{*}-1}\right]\left|\hbar\left(q, \mu^{*}(q)\right)\right| \mathrm{d} q \\
&+\frac{1}{\Gamma\left(\sigma^{*}\right)} \int_{a_{1}}^{a_{2}} \varphi^{\prime}(q)\left(\varphi\left(a_{2}\right)-\varphi(q)\right)^{\sigma^{*}-1}\left|\hbar\left(q, \mu^{*}(q)\right)\right| \mathrm{d} q \\
& \leq \frac{1}{\Gamma\left(\sigma^{*}\right)} \int_{s_{0}}^{a_{1}} \varphi^{\prime}(q)\left[\left(\varphi\left(a_{2}\right)-\varphi(q)\right)^{\sigma^{*}-1}-\left(\varphi\left(a_{1}\right)-\varphi(q)\right)^{\sigma^{*}-1}\right]\left|\psi\left(\mu^{*}(q)\right)\right| \mathrm{d} q \\
&+\frac{1}{\Gamma\left(\sigma^{*}\right)} \int_{a_{1}}^{a_{2}} \varphi^{\prime}(q)\left(\varphi\left(a_{2}\right)-\varphi(q)\right)^{\sigma^{*}-1}\left|\psi\left(\mu^{*}(q)\right)\right| \mathrm{d} q \\
& \times \frac{\left\|m^{*}\right\|}{\Gamma\left(\sigma^{*}+1\right)}\left[-\left(\varphi\left(a_{2}\right)-\varphi\left(a_{1}\right)\right)^{\sigma^{*}}+\left(\varphi\left(a_{2}\right)-\varphi\left(s_{0}\right)\right)^{\sigma^{*}}\right. \\
&\left.-\left(\varphi\left(a_{1}\right)-\varphi\left(s_{0}\right)\right)^{\sigma^{*}}+\left(\varphi\left(a_{2}\right)-\varphi\left(a_{1}\right)\right)^{\sigma^{*}}\right] \\
& \times \frac{\left\|m^{*}\right\|}{\Gamma\left(\sigma^{*}+1\right)}\left[\left(\varphi\left(a_{2}\right)-\varphi\left(s_{0}\right)\right)^{\sigma^{*}}-\left(\varphi\left(a_{1}\right)-\varphi\left(s_{0}\right)\right)^{\sigma^{*}}\right],
\end{aligned}
$$


which implies that $\left|T^{1} \mu^{*}\left(a_{2}\right)-T^{1} \mu^{*}\left(a_{1}\right)\right| \rightarrow 0$ as $a_{1} \rightarrow a_{2}$. Thus $T^{1}$ is equicontinuous and is relatively compact on $V_{\epsilon}$. The operator is now compact due to the application of Arzela-Ascoli theorem. In the last stage, we intend to show that $T^{2}$ is contractive:

$$
\begin{aligned}
\left|T^{2} \mu_{1}^{*}(a)-T^{2} \mu_{2}^{*}(a)\right| & \left|\varphi(a)-\varphi\left(s_{0}\right)\right|\left[\frac{1}{\Gamma\left(\sigma^{*}\right)} \int_{s_{0}}^{M} \varphi^{\prime}(q)(\varphi(M)-\varphi(q))^{\sigma^{*}-1}\left|\hbar\left(q, \mu_{1}^{*}(q)\right)-\hbar\left(q, \mu_{2}^{*}(q)\right)\right| \mathrm{d} q\right. \\
\leq & \left.+\sum_{i=1}^{m} \beta_{i} \frac{1}{\Gamma\left(p_{i}+\sigma^{*}\right)} \int_{s_{0}}^{\xi_{i}} \varphi^{\prime}(q)\left(\varphi\left(\xi_{i}\right)-\varphi(q)\right)^{p_{i}+\sigma^{*}-1}\left|\hbar\left(q, \mu_{1}^{*}(q)\right)-\hbar\left(q, \mu_{2}^{*}(q)\right)\right| \mathrm{d} q\right] \\
\leq & \frac{\left|\varphi(a)-\varphi\left(s_{0}\right)\right|}{|\Lambda|} \\
& \times\left[\frac{1}{\Gamma\left(\sigma^{*}\right)} \int_{s_{0}}^{M} \varphi^{\prime}(q)(\varphi(M)-\varphi(q))^{\sigma^{*}-1}\left|\eta^{*}(q)\right|\left[\psi\left(\left|\mu_{1}^{*}(q)\right|\right)-\psi\left(\left|\mu_{2}^{*}(q)\right|\right)\right] \mathrm{d} q\right. \\
& +\sum_{i=1}^{m} \beta_{i} \frac{1}{\Gamma\left(p_{i}+\sigma^{*}\right)} \\
& \left.\times \int_{s_{0}}^{\xi_{i}} \varphi^{\prime}(q)\left(\varphi\left(\xi_{i}\right)-\varphi(q)\right)^{p_{i}+\sigma^{*}-1}\left|\eta^{*}(q)\right|\left[\psi\left(\left|\mu_{1}^{*}(q)\right|\right)-\psi\left(\left|\mu_{2}^{*}(q)\right|\right)\right] \mathrm{d} q\right] \\
\leq & \frac{\left\|\eta^{*}\right\|\left|\mu_{1}^{*}-\mu_{2}^{*} \| \varphi(a)-\varphi\left(s_{0}\right)\right|}{|\Lambda|}\left[\frac{1}{\Gamma\left(\sigma^{*}\right)} \int_{s_{0}}^{M} \varphi^{\prime}(q)(\varphi(M)-\varphi(q))^{\sigma^{*}-1} \mathrm{~d} q\right. \\
& \left.+\sum_{i=1}^{m} \beta_{i} \frac{1}{\Gamma\left(p_{i}+\sigma^{*}\right)} \int_{s_{0}}^{\xi_{i}} \varphi^{\prime}(q)\left(\varphi\left(\xi_{i}\right)-\varphi(q)\right)^{p_{i}+\sigma^{*}-1} \mathrm{~d} q\right] \\
\leq & \left.\frac{\left\|\eta^{*}\right\|\left(\left|\varphi(M)-\varphi\left(s_{0}\right)\right|\right)^{\sigma^{*}+1}}{|\Lambda| \Gamma\left(\sigma^{*}+1\right)}+\sum_{i=1}^{m} m \beta_{i} \frac{\left\|\eta^{*}\right\|\left(\left|\varphi(M)-\varphi\left(s_{0}\right)\right|\right)^{p_{i}+\sigma^{*}+1}}{|\Lambda| \Gamma\left(p_{i}+\sigma^{*}+1\right)}\right]\left|\mu_{1}^{*}-\mu_{2}^{*}\right| \\
\leq & \Omega_{2}\left|\mu_{1}^{*}-\mu_{2}^{*}\right|,
\end{aligned}
$$

which implies that

$$
\left|T^{2} \mu_{1}^{*}(a)-T^{2} \mu_{2}^{*}(a)\right| \leq \Omega_{2}\left|\mu_{1}^{*}-\mu_{2}^{*}\right| .
$$

Therefore, the operator $T^{2}$ is contractive on $V_{\epsilon}$ with constant $\Omega_{2}<1$. So BVP (1) has at least one solution.

\section{Fractional $\varphi$-BVP (2)}

We will discuss the existence of solutions to problem (2) in this section. We recall a function $\mu^{*} \in C_{N}(J, N)$ is announced as the solution of problem (2) provided that it follows the boundary conditions, and there exists a function $\mu^{*} \in L^{1}(J)$ such that $\mu^{*}(a) \in K\left(a, \mu^{*}(a)\right)$ for almost all $a \in J$ and

$$
\begin{aligned}
\mu^{*}(a)= & \frac{1}{\Gamma\left(\sigma^{*}\right)} \int_{s_{0}}^{a} \varphi^{\prime}(q)(\varphi(a)-\varphi(q))^{\sigma^{*}-1} \mu^{*}(q) \mathrm{d} q \\
& -\frac{\left(\varphi(a)-\varphi\left(s_{0}\right)\right)}{\Lambda}\left[\frac{1}{\Gamma\left(\sigma^{*}\right)} \int_{s_{0}}^{M} \varphi^{\prime}(q)(\varphi(M)-\varphi(q))^{\sigma^{*}-1} \mu^{*}(q) \mathrm{d} q\right.
\end{aligned}
$$




$$
\left.+\sum_{i=1}^{m} \beta_{i} \frac{1}{\Gamma\left(p_{i}+\sigma^{*}\right)} \int_{s_{0}}^{\xi_{i}} \varphi^{\prime}(q)\left(\varphi\left(\xi_{i}\right)-\varphi(q)\right)^{p_{i}+\sigma^{*}-1} \mu^{*}(q) \mathrm{d} q\right]
$$

for all $a \in J$. For each $\mu^{*} \in N$, we demonstrate the selections' set of $K$ by

$$
S_{K, \mu^{*}}=\left\{\mu^{*} \in L^{1}(J): \mu^{*}(a) \in K\left(a, \mu^{*}(a)\right) \text { for all most all } a \in J\right\}
$$

Assume the operator $L: N \rightarrow \mathbb{P}(N)$ by

$$
L\left(\mu^{*}\right)=\left\{r \in N \text { : there exists } \mu^{*} \in S_{K, \mu^{*}} \text { such that } r(a)=\pi(a) \text { for all } a \in J\right\},
$$

where

$$
\begin{aligned}
\pi(a)= & \frac{1}{\Gamma\left(\sigma^{*}\right)} \int_{s_{0}}^{a} \varphi^{\prime}(q)(\varphi(a)-\varphi(q))^{\sigma^{*}-1} \mu^{*}(q) \mathrm{d} q \\
& -\frac{\left(\varphi(a)-\varphi\left(s_{0}\right)\right)}{\Lambda}\left[\frac{1}{\Gamma\left(\sigma^{*}\right)} \int_{s_{0}}^{M} \varphi^{\prime}(q)(\varphi(M)-\varphi(q))^{\sigma^{*}-1} \mu^{*}(q) \mathrm{d} q\right. \\
& \left.+\sum_{i=1}^{m} \beta_{i} \frac{1}{\Gamma\left(p_{i}+\sigma^{*}\right)} \int_{s_{0}}^{\xi_{i}} \varphi^{\prime}(q)\left(\varphi\left(\xi_{i}\right)-\varphi(q)\right)^{p_{i}+\sigma^{*}-1} \mu^{*}(q) \mathrm{d} q\right]
\end{aligned}
$$

Theorem 7 Regard a set-valued map $K: J \times N \rightarrow \mathbb{P}_{\mathbb{C P}}(N)$. Suppose that

$C_{6}$ : The set-valued map $K$ is bounded and integrable with the property that $K\left(\cdot, \mu_{1}^{*}\right)$ : $J \rightarrow \mathbb{P}_{\mathbb{C P}}(N)$ is measurable for $\mu_{1}^{*} \in N$.

$C_{7}$ : There are a member $\omega \in C(J,[0, \infty))$ and a map $\psi \in \Psi$ such that

$$
\mathbb{H}_{\mathrm{d}}\left(K\left(a, \mu_{1}^{*}\right), K\left(a, \mu_{1}^{*}\right)\right) \leq \frac{\omega(a) \lambda^{*}}{\|\omega\|} \psi\left(\left|\mu_{1}^{*}-\mu_{1}^{*}\right|\right)
$$

for all $a \in J$ and $\mu_{1}^{*}, \mu_{1}^{*} \in N$, where $\lambda^{*}=\frac{1}{\Omega_{1}}$.

$C_{8}$ : There exists a function $G: \mathbb{R}^{2} \times \mathbb{R}^{2} \rightarrow \mathbb{R}$ such that $G\left(\left(\mu_{1}^{*}, \mu_{1}^{*}\right)\right) \geq 0$ for all $\mu_{1}^{*}, \mu_{1}^{*} \in N$.

$C_{9}$ : A sequence $\left\{\mu_{n}^{*}\right\}$ of points of $N$ such that $\mu_{n}^{*} \rightarrow \mu^{*}$ and

$$
G\left(\left(\mu_{n}^{*}(a), \mu_{n+1}^{*}(a)\right)\right) \geq 0
$$

for all $a \in J, n \geq 1$, there exists a subsequence $\left\{\mu_{n_{j}}^{*}\right\}$ of $\left\{\mu_{n}^{*}\right\}$ with

$$
G\left(\left(\mu_{n_{j}}^{*}(a), \mu^{*}(a)\right)\right) \geq 0
$$

for all $a \in J$ and $1 \leq j$.

$C_{10}$ : There are elements $\mu_{0}^{*}$ of $N$ and $r$ of $L\left(\mu_{0}^{*}\right)$ with

$$
G\left(\left(\mu_{0}^{*}(a), r(a)\right)\right) \geq 0
$$

for all $a \in J$, where the operator $L: N \rightarrow \mathbb{P}(N)$.

$C_{11}$ : For every member $\mu^{*}$ of $N$ and $r$ of $L\left(\mu^{*}\right)$ such that

$$
G\left(\left(\mu^{*}(a), r(a)\right)\right) \geq 0
$$


an element $w^{*}$ of $L\left(\mu^{*}\right)$ exists such that

$$
G\left(\left(r(a), w^{*}(a)\right)\right) \geq 0
$$

for all $a \in J$. Then BVP (2) has a solution.

Proof Eventually, the point fixed by the map $L$ is characterized as a solution of BVP (2). For all $\mu^{*} \in N$, the set-valued map $a \mapsto K\left(a, \mu^{*}(a)\right)$ is closed-valued as well as measurable, so there is a selection of the map $K$ which is measurable and the set $S_{K, \mu^{*}}$ is nonempty. We would prove that $L\left(\mu^{*}\right)$ is closed as a subset of $N$ for all $\mu^{*} \in N$. Assume a convergent sequence $\left\{\mu_{n}^{*}\right\}$ of points of $L\left(\mu^{*}\right)$ tending to $\mu^{*}$. Corresponding to every $n$, an element $\mu_{n}^{*} \in S_{K, \mu^{*}}$ exists such that

$$
\begin{aligned}
\mu_{n}^{*}(a)= & \frac{1}{\Gamma\left(\sigma^{*}\right)} \int_{s_{0}}^{a} \varphi^{\prime}(q)(\varphi(a)-\varphi(q))^{\sigma^{*}-1} \Upsilon_{n}(q) \mathrm{d} q \\
& -\frac{\left(\varphi(a)-\varphi\left(s_{0}\right)\right)}{\Lambda}\left[\frac{1}{\Gamma\left(\sigma^{*}\right)} \int_{s_{0}}^{M} \varphi^{\prime}(q)(\varphi(M)-\varphi(q))^{\sigma^{*}-1} \Upsilon_{n}(q) \mathrm{d} q\right. \\
& \left.+\sum_{i=1}^{m} \beta_{i} \frac{1}{\Gamma\left(p_{i}+\sigma^{*}\right)} \int_{s_{0}}^{\xi_{i}} \varphi^{\prime}(q)\left(\varphi\left(\xi_{i}\right)-\varphi(q)\right)^{p_{i}+\sigma^{*}-1} \Upsilon_{n}(q) \mathrm{d} q\right]
\end{aligned}
$$

for all $a \in J$. Since the values of $K$ are compact, on the basis of this observation, we consider a subsequence of $\left\{\mu_{n}^{*}\right\}$ that tends to $\mu^{*}$ in $L^{1}(J)$. Hence, $\mu_{n}^{*} \in S_{K, \mu^{*}}$ and

$$
\begin{aligned}
\mu_{n}^{*}(a) \rightarrow \mu^{*}(a)= & \frac{1}{\Gamma\left(\sigma^{*}\right)} \int_{s_{0}}^{a} \varphi^{\prime}(q)(\varphi(a)-\varphi(q))^{\sigma^{*}-1} \Upsilon(q) \mathrm{d} q \\
& -\frac{\left(\varphi(a)-\varphi\left(s_{0}\right)\right)}{\Lambda}\left[\frac{1}{\Gamma\left(\sigma^{*}\right)} \int_{s_{0}}^{M} \varphi^{\prime}(q)(\varphi(M)-\varphi(q))^{\sigma^{*}-1} \Upsilon(q) \mathrm{d} q\right. \\
& \left.+\sum_{i=1}^{m} \beta_{i} \frac{1}{\Gamma\left(p_{i}+\sigma^{*}\right)} \int_{s_{0}}^{\xi_{i}} \varphi^{\prime}(q)\left(\varphi\left(\xi_{i}\right)-\varphi(q)\right)^{p_{i}+\sigma^{*}-1} \Upsilon(q) \mathrm{d} q\right]
\end{aligned}
$$

for all $a \in J$, which implies $\mu^{*} \in L\left(\mu^{*}\right)$, and so the values of $L$ are closed. Since the setvalued map $K$ is compact-valued as well, it is indeed simple to demonstrate that $L\left(\mu^{*}\right)$ is bounded for all $\mu^{*} \in N$. We have to show that the set-valued map $L$ is $\alpha-\psi$-contractive. To do this, we regard

$$
\alpha\left(\mu^{*}, \mu^{*}\right)= \begin{cases}1 & \text { if } G\left(\left(\mu^{*}(a), \mu^{*}(a)\right)\right) \geq 0 \\ 0 & \text { otherwise }\end{cases}
$$

for all $\mu^{*}, \mu^{*} \in N$ and $r_{1}^{*} \in L\left(\mu^{*}\right)$, choose $\mu_{1}^{*} \in S_{K, \mu^{*}}$ such that

$$
\begin{aligned}
r_{1}^{*}= & \frac{1}{\Gamma\left(\sigma^{*}\right)} \int_{s_{0}}^{a} \varphi^{\prime}(q)(\varphi(a)-\varphi(q))^{\sigma^{*}-1} \Upsilon_{1}(q) \mathrm{d} q \\
& -\frac{\left(\varphi(a)-\varphi\left(s_{0}\right)\right)}{\Lambda}\left[\frac{1}{\Gamma\left(\sigma^{*}\right)} \int_{s_{0}}^{M} \varphi^{\prime}(q)(\varphi(M)-\varphi(q))^{\sigma^{*}-1} \Upsilon_{1}(q) \mathrm{d} q\right.
\end{aligned}
$$




$$
\left.+\sum_{i=1}^{m} \beta_{i} \frac{1}{\Gamma\left(p_{i}+\sigma^{*}\right)} \int_{s_{0}}^{\xi_{i}} \varphi^{\prime}(q)\left(\varphi\left(\xi_{i}\right)-\varphi(q)\right)^{p_{i}+\sigma^{*}-1} \Upsilon_{1}(q) \mathrm{d} q\right]
$$

for all $a \in J$.

$$
\mathbb{H}_{d}\left(K\left(a, \mu^{*}(a)\right), K\left(a, \mu^{*}(a)\right) \leq \frac{\omega(a) \lambda^{*}}{\|\omega\|} \psi\left(\left|\mu_{1}^{*}-\mu_{1}^{*}\right|\right)\right.
$$

for all $\mu^{*}, \mu^{*} \in N$ with $G\left(\left(\mu^{*}(a), \mu^{*}(a)\right)\right) \geq 0$ for all $a \in J$. Therefore, an element $\pi \in$ $K\left(a, \mu^{*}(a)\right)$ exists such that

$$
\left|\mu_{1}^{*}(a)-\pi\right| \leq \frac{\omega(a) \lambda^{*}}{\|\omega\|} \psi\left(\left|\mu_{1}^{*}-\mu_{1}^{*}\right|\right) .
$$

Now assume a set-valued map $B^{*}: J \rightarrow \mathbb{P}(N)$ which is given as follows:

$$
B^{*}(a)=\left\{\pi \in N:\left|\mu_{1}^{*}-\pi(a)\right| \leq \frac{\omega(a) \lambda^{*}}{\|\omega\|} \psi\left(\left|\mu_{1}^{*}-\mu_{1}^{*}\right|\right)\right\}
$$

for all $a \in J$. Notice that the set-valued map $B^{*}(\cdot) \cap K\left(a, \mu^{*}(a)\right)$ is measurable because $\mu_{1}^{*}$ and $\tau=\frac{\omega(a) \lambda^{*}}{\|\omega\|} \psi\left(\left|\mu_{1}^{*}-\mu_{1}^{*}\right|\right)$ are measurable. Now let $\mu_{2}^{*} \in K\left(a, \mu^{*}(a)\right)$

$$
\left|\mu_{1}^{*}(a)-\mu_{2}^{*}(a)\right| \leq \frac{\omega(a) \lambda^{*}}{\|\omega\|} \psi\left(\left|\mu_{1}^{*}-\mu_{1}^{*}\right|\right)
$$

for all $a \in J$. Let us define $r_{2}^{*} \in L(a)$ by

$$
\begin{aligned}
r_{2}^{*}(a)= & \frac{1}{\Gamma\left(\sigma^{*}\right)} \int_{s_{0}}^{a} \varphi^{\prime}(q)(\varphi(a)-\varphi(q))^{\sigma^{*}-1} \Upsilon_{2}(q) \mathrm{d} q \\
& -\frac{\left(\varphi(a)-\varphi\left(s_{0}\right)\right)}{\Lambda}\left[\frac{1}{\Gamma\left(\sigma^{*}\right)} \int_{s_{0}}^{M} \varphi^{\prime}(q)(\varphi(M)-\varphi(q))^{\sigma^{*}-1} \Upsilon_{2}(q) \mathrm{d} q\right. \\
& \left.+\sum_{i=1}^{m} \beta_{i} \frac{1}{\Gamma\left(p_{i}+\sigma^{*}\right)} \int_{s_{0}}^{\xi_{i}} \varphi^{\prime}(q)\left(\varphi\left(\xi_{i}\right)-\varphi(q)\right)^{p_{i}+\sigma^{*}-1} \Upsilon_{2}(q) \mathrm{d} q\right]
\end{aligned}
$$

for all $a \in J$. Let $\sup _{a \in J}|\omega(a)|=\|\omega\|$, then

$$
\begin{aligned}
\left|r_{1}^{*}-r_{2}^{*}\right| \leq & \frac{1}{\Gamma\left(\sigma^{*}\right)} \int_{s_{0}}^{a} \varphi^{\prime}(q)(\varphi(a)-\varphi(q))^{\sigma^{*}-1}\left|\Upsilon_{1}(q)-\Upsilon_{2}(q)\right| \mathrm{d} q \\
& -\frac{\left(\varphi(a)-\varphi\left(s_{0}\right)\right)}{\Lambda}\left[\frac{1}{\Gamma\left(\sigma^{*}\right)} \int_{s_{0}}^{M} \varphi^{\prime}(q)(\varphi(M)-\varphi(q))^{\sigma^{*}-1}\left|\Upsilon_{1}(q)-\Upsilon_{2}(q)\right| \mathrm{d} q\right. \\
& \left.+\sum_{i=1}^{m} \beta_{i} \frac{1}{\Gamma\left(p_{i}+\sigma^{*}\right)} \int_{s_{0}}^{\xi_{i}} \varphi^{\prime}(q)\left(\varphi\left(\xi_{i}\right)-\varphi(q)\right)^{p_{i}+\sigma^{*}-1}\left|\Upsilon_{1}(q)-\Upsilon_{2}(q)\right| \mathrm{d} q\right] \\
\leq & \frac{\left(\left|\varphi(M)-\varphi\left(s_{0}\right)\right|\right)^{\sigma^{*}}}{\Gamma\left(\sigma^{*}+1\right)}\|\omega\| \psi\left(\left\|\mu^{*}-\mu^{*}\right\|\right) \frac{\lambda^{*}}{\|\omega\|} \\
& +\frac{\left(\left|\left(\varphi(M)-\varphi\left(s_{0}\right)\right)\right|\right)^{\sigma^{*}+1}}{|\Lambda| \Gamma\left(\sigma^{*}+1\right)}\|\omega\| \psi\left(\left\|\mu^{*}-\mu^{*}\right\|\right) \frac{\lambda^{*}}{\|\omega\|}
\end{aligned}
$$




$$
\begin{aligned}
& +\sum_{i=1}^{m} \beta_{i} \frac{m\left(\left|\left(\varphi(M)-\varphi\left(s_{0}\right)\right)\right|\right)^{p_{i}+\sigma^{*}+1}}{|\Lambda| \Gamma\left(p_{i}+\sigma^{*}+1\right)}\|\omega\| \psi\left(\left\|\mu^{*}-\mu^{*}\right\|\right) \frac{\lambda^{*}}{\|\omega\|} \\
= & {\left[\frac{\left(\left|\varphi(M)-\varphi\left(s_{0}\right)\right|\right)^{\sigma^{*}}}{\Gamma\left(\sigma^{*}+1\right)}+\frac{\left(\left|\left(\varphi(M)-\varphi\left(s_{0}\right)\right)\right|\right)^{\sigma^{*}+1}}{|\Lambda| \Gamma\left(\sigma^{*}+1\right)}\right.} \\
& \left.+\sum_{i=1}^{m} \beta_{i} \frac{m\left(\left|\left(\varphi(M)-\varphi\left(s_{0}\right)\right)\right|\right)^{p_{i}+\sigma^{*}+1}}{|\Lambda| \Gamma\left(p_{i}+\sigma^{*}+1\right)}\right]\|\omega\| \psi\left(\left\|\mu^{*}-\mu^{*}\right\|\right) \frac{\lambda^{*}}{\|\omega\|} \\
= & \Omega_{1}\|\omega\| \psi\left(\left\|\mu^{*}-\mu^{*}\right\|\right) \frac{\lambda^{*}}{\|\omega\|} \\
= & \Omega_{1} \lambda^{*} \psi\left(\left\|\mu^{*}-\mu^{*}\right\|\right) .
\end{aligned}
$$

Thus $\alpha\left(\mu^{*}, \mu^{*}\right) \mathbb{H}_{\mathrm{d}}\left(L\left(\mu^{*}\right), L\left(\mu^{*}\right)\right) \leq \psi\left(\left\|\mu^{*}-\mu^{*}\right\|\right)$ holds for all $\mu^{*}, \mu^{*} \in N$, which implies that $L\left(\mu^{*}\right)$ is an $\alpha-\psi$-contractive set-valued mapping. Now, let $\mu^{*} \in N$ and $\mu^{*} \in L$ be two functions such that $\alpha\left(\mu^{*}, \mu^{*}\right) \geq 1$. In this case,

$$
G\left(\left(\mu^{*}(a), \mu^{*}(a)\right)\right) \geq 0
$$

So, there exists a function $\pi \in L\left(\mu^{\prime *}\right)$ such that

$$
G\left(\left(\mu^{*}(a), \pi(a)\right)\right) \geq 0
$$

This follows that $\alpha\left(\mu^{*}, \pi\right) \geq 1$, which implies that the operator $L$ is $\alpha$-admissible. Now, suppose that $\mu_{0}^{*} \in N$ and $\mu^{*} \in L\left(\mu_{0}^{*}\right)$ are such that

$$
G\left(\left(\mu_{0}^{*}(a), \mu^{*}(a)\right)\right) \geq 0
$$

for all $a \in J$. Then we have $\alpha\left(\mu_{0}^{*}, \mu^{*}\right) \geq 1$. Let $\left\{\mu_{n}^{*}\right\}$ be a sequence in $N$ such that $\mu_{n}^{*} \rightarrow \mu^{*}$ and $\alpha\left(\mu_{n}^{*}, \mu_{n+1}^{*}\right) \geq 1$ for all $n$. Then we get

$$
G\left(\left(\mu_{n}^{*}(a), \mu_{n+1}^{*}(a)\right)\right) \geq 0
$$

By using $C_{9}$ there exists a subsequence $\left\{\mu_{n_{j}}^{*}\right\}$ of $\left\{\mu_{n}^{*}\right\}$ such that

$$
G\left(\left(\mu_{n_{j}}^{*}(a), \mu^{*}(a)\right)\right) \geq 0
$$

for all $a \in J$. Thus $\alpha\left(\mu_{n}^{*}, \mu^{*}\right) \geq 1$ for all $j$, which implies that the Banach space $N$ admits $\left(\mathbf{P}_{2}\right)$. As a conclusion, Theorem 4 is proved, therefore the mapping $L$ admits a fixed point which is the solution for BVP (2).

Theorem 8 Let $K: J \times N \rightarrow \mathbb{P}(N)$ be a set-valued mapping. Assume that

$C_{12}$ : There exists a nonnegative function $\psi:[0, \infty) \rightarrow[0, \infty)$ which is nondecreasing upper semi-continuous such that $\liminf _{\rightarrow \infty}(a-\psi(a))>0$ and $\psi(a)<$ a for all $a>0$.

$C_{13}$ : The multifunction $K: J \times N \rightarrow \mathbb{P}(N)$ is bounded and integrable with $K\left(\cdot, \mu_{1^{\prime}}^{*}, \mu_{2^{\prime}}^{*}\right)$ : $J \rightarrow \mathbb{P}(N)$ is measurable for all $\mu_{1}^{*}, \mu_{2}^{*} \in N$. 
$C_{14}$ : There exists a nonnegative function $\omega \in C(J,[0, \infty))$ such that

$$
\mathbb{H}_{\mathrm{d}}^{*}\left(K\left(a, \mu_{1}^{*}\right)-K\left(a, \mu^{*}{ }_{1}\right)\right) \leq \omega(a) \lambda^{*} \psi\left(\left|\mu_{1}^{*}-\mu^{*}{ }_{1}\right|\right)
$$

for all $\mu_{1}^{*}, \mu^{*}{ }_{1} \in N$.

$C_{15}$ : L admits the approximate endpoint property. Then there exists a solution to BVP (2).

Proof We ought to prove that the set-valued mapping $L: N \rightarrow \mathbb{P}(N)$ has an endpoint. In the beginning, we have to make sure that $L\left(\mu^{*}\right)$ is closed for all $\mu^{*} \in N$. Since the map $a \mapsto$ $K\left(a, \mu^{*}(a)\right)$ is measurable and is closed-valued for all $\mu^{*} \in N$, the map $K$ has a measurable selection, and so $S_{K, \mu^{*}} \neq \emptyset$ for all $\mu^{*} \in N$. Following the method given in Theorem 7, we can easily show that $L\left(\mu^{*}\right)$ is closed. Moreover, the boundedness of $L\left(\mu^{*}\right)$ can be verified since the map $K$ is compact. At last, we can easily observe that the inequality $\mathbb{H}_{\mathrm{d}}\left(L\left(\mu^{*}\right), L(\pi)\right) \leq$ $\psi\left(\left\|\mu^{*}-\pi\right\|\right)$ is satisfied. Suppose that $\mu^{*}, \pi \in N$ and $r_{1}^{*} \in L(\pi)$. Choose $\mu_{1}^{*} \in S_{K, \pi}$ such that

$$
\begin{aligned}
r_{1}^{*}= & \frac{1}{\Gamma\left(\sigma^{*}\right)} \int_{s_{0}}^{a} \varphi^{\prime}(q)(\varphi(a)-\varphi(q))^{\sigma^{*}-1} \Upsilon_{1}(q) \mathrm{d} q \\
& -\frac{\left(\varphi(a)-\varphi\left(s_{0}\right)\right)}{\Lambda}\left[\frac{1}{\Gamma\left(\sigma^{*}\right)} \int_{s_{0}}^{M} \varphi^{\prime}(q)(\varphi(M)-\varphi(q))^{\sigma^{*}-1} \Upsilon_{1}(q) \mathrm{d} q\right. \\
& \left.+\sum_{i=1}^{m} \beta_{i} \frac{1}{\Gamma\left(p_{i}+\sigma^{*}\right)} \int_{s_{0}}^{\xi_{i}} \varphi^{\prime}(q)\left(\varphi\left(\xi_{i}\right)-\varphi(q)\right)^{p_{i}+\sigma^{*}-1} \Upsilon_{1}(q) \mathrm{d} q\right]
\end{aligned}
$$

for all $a \in J$. Therefore,

$$
\mathbb{H}_{\mathrm{d}}\left(K\left(a, \mu^{*}\right)-K(a, \pi(a))\right) \leq \omega(a) \lambda^{*} \psi\left(\left|\mu^{*}-\pi\right|\right)
$$

for all $a \in J$, there exists $\varpi^{*} \in K\left(a, \mu^{*}(a)\right)$ such that

$$
\left|\mu_{1}^{*}(a)-\varpi^{*}\right| \leq \omega \lambda^{*} \psi\left(\left|\mu^{*}-\pi\right|\right) .
$$

Assuming a set-valued mapping $O^{*}: J \rightarrow \mathbb{P}(N)$ defined by

$$
O^{*}(a)=\left\{\mu^{*} \in N:\left|\Upsilon_{1}-\Upsilon(a)\right| \leq \omega(a) \lambda^{*} \psi\left(\left|\mu^{*}(a)-\pi(a)\right|\right)\right\}
$$

The map $\mu_{1}^{*}$ is measurable and $\varphi^{*}=\omega(a) \lambda^{*} \psi\left(\left|\mu^{*}-\pi\right|\right)$, therefore, the set-valued map $O^{*}(\cdot) \cap K\left(a, \mu^{*}(a)\right)$ is measurable. Now we choose $\mu_{2}^{*} \in K\left(a, \mu^{*}(a)\right)$ such that

$$
\left|\Upsilon_{1}(a)-\Upsilon_{2}(a)\right| \leq \omega(a) \frac{1}{\Omega_{1}} \psi\left(\left|\mu^{*}-\pi\right|\right)
$$

for all $a \in J$. Select $r_{2}^{*} \in L\left(\mu^{*}\right)$ such that

$$
\begin{aligned}
r_{2}^{*}(a)= & \frac{1}{\Gamma\left(\sigma^{*}\right)} \int_{s_{0}}^{a} \varphi^{\prime}(q)(\varphi(a)-\varphi(q))^{\sigma^{*}-1} \Upsilon_{2}(q) \mathrm{d} q \\
& -\frac{\left(\varphi(a)-\varphi\left(s_{0}\right)\right)}{\Lambda}\left[\frac{1}{\Gamma\left(\sigma^{*}\right)} \int_{s_{0}}^{M} \varphi^{\prime}(q)(\varphi(M)-\varphi(q))^{\sigma^{*}-1} \Upsilon_{2}(q) \mathrm{d} q\right.
\end{aligned}
$$




$$
\left.+\sum_{i=1}^{m} \beta_{i} \frac{1}{\Gamma\left(p_{i}+\sigma^{*}\right)} \int_{s_{0}}^{\xi_{i}} \varphi^{\prime}(q)\left(\varphi\left(\xi_{i}\right)-\varphi(q)\right)^{p_{i}+\sigma^{*}-1} \Upsilon_{2}(q) \mathrm{d} q\right]
$$

for all $a \in J$. On the lines of Theorem 8 , we get

$$
\begin{aligned}
\left\|r_{1}^{*}-r_{2}^{*}\right\| & =\sup _{a \in J}\left|r_{1}^{*}(a)-r_{2}^{*}(a)\right| \\
& \leq\left(\Omega_{1}\right) \lambda^{*} \psi\left(\left\|\mu^{*}-\mu^{*}\right\|\right) \\
& =\psi\left(\left\|\mu^{*}-\mu^{\prime}\right\|\right) .
\end{aligned}
$$

Hence, $\mathbb{H}_{\mathrm{d}}\left(L\left(\mu^{*}\right), L(\pi)\right) \leq \psi\left(\left\|\mu^{*}-\mu^{*}\right\|\right)$ for all $\mu^{*}, \mu^{*^{\prime}} \in N$. By using $\left(C_{15}\right)$ one can easily find that $L$ has the endpoint property. Now, by using Theorem 4 , there exists $\mu^{*} \in N$ such that $L\left(\mu^{*}\right)=\left\{\mu^{*}\right\}$. This implies that $\mu^{*}$ is a solution of BVP (2).

\section{Examples}

Example 1 Consider the boundary value problem

$$
\left\{\begin{array}{l}
{ }^{C} D_{0}^{1.79, \exp (a+1)} \mu^{*}(a)=\frac{a \cos (a)\left|\mu^{*}(a)\right|}{340\left(\left|\mu^{*}(a)\right|+1\right)}, \quad a \in[0,1] \\
\mu^{*}(0)=0, \\
\mu^{*}(1)=0.71^{R L} I_{0}^{1.69, \exp (a+1)} \mu^{*}(0.37)+0.85^{R L} I_{0}^{1.93, \exp (a+1)} \mu^{*}(0.39),
\end{array}\right.
$$

where $a \in[0,1], \sigma^{*}=1.79, s_{0}=0, M=1, \mathcal{M}=2, p_{1}=1.69, p_{2}=1.93, \xi_{1}=0.37, \xi_{2}=0.39$, $\beta_{1}=0.71, \beta_{2}=0.85$. Here, ${ }^{C} D_{0}^{1.79, \exp (a+1)}$ denotes the fractional derivative of the Caputo

Figure 1 Graph of the function $\hbar\left(a, \mu^{*}\right)$

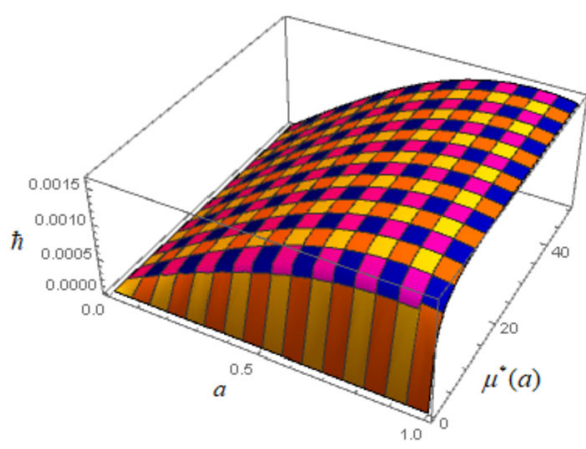

Figure 2 Graph of the function $\frac{a}{340} \psi\left(\left|\mu^{*}\right|\right)$

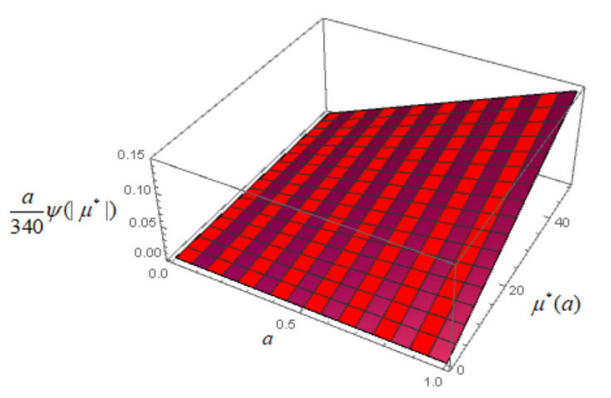


Figure 3 Comparison of the inequality $\hbar\left(a, \mu^{*}\right)$ $\leq \frac{a}{340} \psi\left(\left|\mu^{*}\right|\right)$

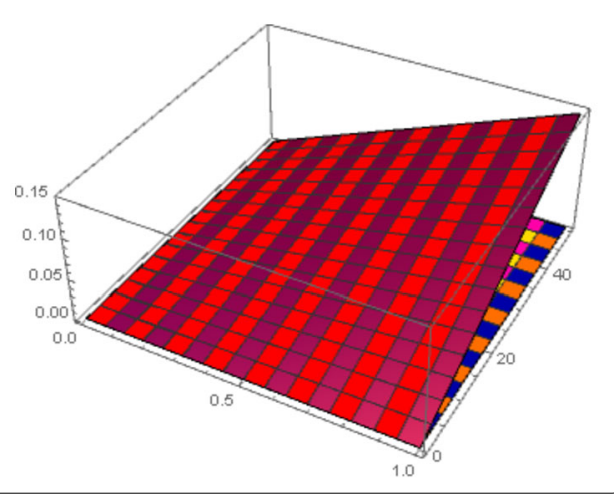

type of order 1.79 and ${ }^{R L} I_{0}^{p_{i}}$ denotes the fractional integral of the Riemann-Liouville type of order $p_{i}$. Let the continuous mapping be defined by $\hbar\left(a, \mu^{*}(a)\right)=\frac{a \cos (a)\left|\mu^{*}(a)\right|}{340\left(\left|\mu^{*}(a)\right|+1\right)}$, we have

$$
\begin{aligned}
& \left|\hbar\left(a, \mu_{1}^{*}(a)\right)-\hbar\left(a, \mu_{2}^{*}(a)\right)\right| \\
& \quad \leq \frac{a}{340}\left|\mu_{1}-\mu_{2}^{*}\right| .
\end{aligned}
$$

Put $\eta^{*}(a)=\frac{a}{340}$ for all $a \in[0,1]$, then $\left\|\eta^{*}\right\|=0.00295$. Consider the continuous and nondecreasing function $\psi:[0 ; 1] \rightarrow \mathbb{R}^{+}$defined by $\psi(a)=a$ for all $a \in \mathbb{R}^{+}$. Then we have $\hbar^{*}\left(a, \mu^{*}(a)\right) \leq \frac{a}{340} \psi\left(\left|\mu^{*}\right|\right)$ (see the comparison of Fig. 1 and Fig. 2 which is presented in Fig. 3). Then we have $\Lambda=1.6094 \Omega_{1}=5.0139, \Omega_{2}=0.0098<1$. Now, by using Theorem 6 , BVP has a solution.

Example 2 Consider the boundary value problem

$$
\left\{\begin{array}{l}
{ }^{C} D_{0}^{1.3, \exp (a / 2)} \mu^{*}(a) \in\left[0, \frac{\exp (\sqrt[3]{a}+1)}{30}+\frac{\sqrt{\pi} \cos (\operatorname{coth}(a))}{2+\exp (a)}+\frac{a \sin (a)\left|\mu^{*}(a)\right|}{126(a+7)}\right], \quad a \in[0,3] \\
\mu^{*}(0)=0, \\
\mu^{*}(3)=0.69^{R L} I_{0}^{1.37, \exp (2 a)} \mu^{*}(0.37)+0.72^{R L} I_{0}^{1.82, \exp (2 a)} \mu^{*}(0.38),
\end{array}\right.
$$

where $\sigma^{*}=1.3, s_{0}=0, M=3, \mathcal{M}=2, p_{1}=1.37, p_{2}=1.82, \xi_{1}=0.37, \xi_{2}=0.38, \beta_{1}=0.69$, $\beta_{2}=0.72$. Here, ${ }^{C} D_{0}^{1.3, \exp (a / 2)}$ denotes the fractional derivative of the Caputo type of order 0.3 and ${ }^{R L} I_{0}^{1.37, \exp (a / 2)}{ }^{R L} I_{0}^{1.82, \exp (a / 2)}$ denotes the fractional integrals of the RiemannLiouville type of orders 1.37 and 1.82. Let the continuous set-valued mapping $K:[0,3] \times$ $\mathbb{R} \rightarrow \mathbb{P}(\mathbb{R})$ be defined by $K\left(a, \mu^{*}(a)\right)=\left[a, \frac{\exp (\sqrt[3]{a}+1)}{30}+\frac{\sqrt{\pi} \cos (\operatorname{coth}(a))}{2+\exp (a)}+\frac{a \sin (a)\left|\mu^{*}(a)\right|}{126(a+7)}\right]$. For $\mu_{1}^{*}, \mu_{2}^{*} \in$ $\mathbb{R}$, we have

$$
\begin{aligned}
\mathbb{H} & \left(K\left(a, \mu_{1}^{*}(a)\right)-K\left(a, \mu_{2}^{*}(a)\right)\right) \\
& \leq \frac{a}{63} \frac{1}{2}\left[\left|\sin \left(\mu_{1}^{*}(a)\right)-\sin \left(\mu_{2}^{*}(a)\right)\right|\right] \\
& \leq \frac{a}{63} \psi\left(\left|\mu_{1}^{*}(a)-\mu_{2}^{*}(a)\right|\right) \\
& \leq \vartheta(a) \psi\left(\left|\mu_{1}^{*}(a)-\mu_{2}^{*}(a)\right|\right) \frac{1}{\Omega_{1}^{*}} .
\end{aligned}
$$


Choose the nonnegative function $\omega \in C([0,3],[0, \infty))$ defined by $\omega(a)=\frac{a}{63}$ for all $a \in[0,3]$. Then $\|\omega\|=\frac{3}{63}=0.047$. Also, consider the nonnegative and nondecreasing upper semicontinuous function $\psi:[0,3) \rightarrow[0,3)$ defined by $\psi(a)=\frac{a}{2}$ for almost all $a>0$. It is clear that $\lim _{a \rightarrow \infty} \inf (a-\psi(a))>0$ and $\psi(a)<a$ for all $a>0$. Then we have $\mathbb{H}_{d}\left(K\left(a, \mu_{1}^{*}(a)\right)-\right.$ $\left.K\left(a, \mu_{2}^{*}(a)\right)\right) \leq \omega(a) \psi\left(\left|\mu_{1}^{*}(a)-\mu_{2}^{*}(a)\right|\right) \frac{1}{\Omega_{1}}$. Then we have $\Omega_{1}=27.2258, \Lambda=3.4493, \lambda^{*}=$ 0.0367 . Consider the operator $L: N \rightarrow \mathbb{P}(N)$ by

$$
L\left(\mu^{*}\right)=\left\{r \in N \text { : there exists } \mu^{*} \in S_{K, \mu^{*}} \text { such that } r(a)=\pi(a) \text { for all } a \in[0,3]\right\},
$$

where

$$
\begin{aligned}
\pi(a)= & \frac{1}{\Gamma(1.3)} \int_{0}^{a} \varphi^{\prime}(q)(\varphi(a)-\varphi(q))^{0,3} \Upsilon(q) \mathrm{d} q \\
& -\frac{(\varphi(a)-1)}{3.4493}\left[\frac{1}{\Gamma(1.3)} \int_{0}^{3} \varphi^{\prime}(q)(\varphi(M)-\varphi(q))^{0.3} \Upsilon(q) \mathrm{d} q\right. \\
& \left.+\sum_{0}^{2} \beta_{i} \frac{1}{\Gamma\left(p_{i}+1.3\right)} \int_{0}^{\xi_{i}} \varphi^{\prime}(q)\left(\varphi\left(\xi_{i}\right)-\varphi(q)\right)^{p_{i}+0.3} \mu^{*}(q) \mathrm{d} q\right] .
\end{aligned}
$$

Now, by using Theorem 8 , BVP has a solution.

\section{Conclusion}

A number of natural phenomena emerging in science and technology are modeled by fractional differential equations (FDEs). The present study deals with a novel class of $\varphi$-CF nonlocal BVP in the fractional $\varphi$-Caputo sense equipped with $\varphi$-RLF-integral nonlocal boundary conditions. We considered the class of admissible mappings w.r.t. to the mapping $\alpha$ equipped with a nondecreasing control function and proved the solutions' existence of fractional $\varphi$-BVPs (1) and (2). The requisite criteria for the existence of solution corresponding to the inclusion version of $\varphi$-BVP have been explored utilizing the AEPproperty. Finally, several simulative examples have been developed to validate research findings. Indeed, one can consider more complex FDEs and FDIs involving $\varphi$-Caputo fractional derivative and even some other fractional operators with the variety of boundary conditions and can prove the solutions' existence using fixed point theory and functional analysis.

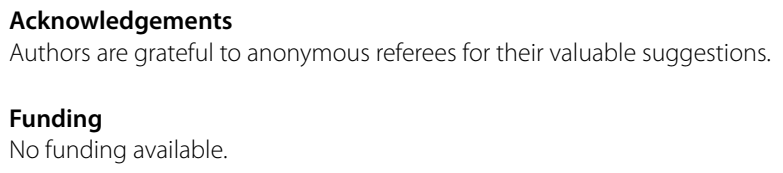




\section{Publisher's Note}

Springer Nature remains neutral with regard to jurisdictional claims in published maps and institutional affiliations.

\section{Received: 11 May 2021 Accepted: 7 July 2021 Published online: 28 July 2021}

\section{References}

1. Atangana, A.: Derivative with a New Parameter: Theory, Methods and Applications. Academic Press, San Diego (2015)

2. Atangana, A.: Fractional Operators with Constant and Variable Order with Application to Geo-Hydrology. Academic Press, San Diego (2017)

3. Atangana, A., Goufo, E.F.D.: Cauchy problems with fractal-fractional operators and applications to groundwater dynamics. Fractals 28(8), 2040043 (2020)

4. Kilbas, A., Srivastava, H., Trujillo, J.: Theory and Applications of Fractional Differential Equations. North-Holland Mathematics Studies, vol. 204 (2006)

5. Mainardi, F.: Fractional Calculus and Waves in Linear Viscoelasticity: An Introduction to Mathematical Models. World Scientific, Singapore (2010)

6. Pratap, A., Raja, R., Alzabut, J., Dianavinnarasi, J., Cao, J., Rajchakit, G.: Finite-time Mittag-Leffler stability of fractional-order quaternion-valued memristive neural networks with impulses. Neural Processing Letters 51(2), $1485-1526(2020)$

7. Alqahtani, B., Aydi, H., Karapinar, E., Rakocevic, V.: A solution for Volterra fractional integral equations by hybrid contractions. Mathematics 7(8), 694 (2019)

8. Karapinar, E., Fulga, A., Rashid, M., Shahid, L., Aydi, H.: Large contractions on quasi-metric spaces with an application to nonlinear fractional differential equations. Mathematics 7(5), 444 (2019)

9. Adigüzel, R.S., Aksoy, Ü, Karapinar, E.: Uniqueness of solution for higher-order nonlinear fractional differential equations with multi-point and integral boundary conditions. Rev. R. Acad. Cienc. Exactas Fís. Nat., Ser. A Mat. 115 155 (2021). https://doi.org/10.1007/s13398-021-01095-3

10. Adigüzel, R.S., Aksoy, Ü., Karapinar, E., Erhan, I.M.: On the solution of a boundary value problem associated with a fractional differential equation. Math. Methods Appl. Sci., 1-12 (2020). https://doi.org/10.1002/mma.6652

11. Ahmad, B., Ntouyas, S.K., Tariboon, J.: On hybrid Caputo fractional integro-differential inclusions with nonlocal conditions. J. Nonlinear Sci. Appl. 9, 4235-4246 (2016)

12. Alsaedi, A., Baleanu, D., Etemad, S., Rezapour, Sh.: On coupled systems of times-fractional differential problems by using a new fractional derivative. J. Funct. Spaces 2016, Article ID 4626940 (2016)

13. Butt, R.I., Abdeljawad, T., Alqudah, M.A., Rehman, M.: Ulam stability of Caputo q-fractional delay difference equation: q-fractional Gronwall inequality approach. J. Inequal. Appl. 2019, 305 (2019)

14. Etemad, S., Ntouyas, S.K., Tariboon, J.: Existence results for three-point boundary value problems for nonlinear fractional differential equations. J. Nonlinear Sci. Appl. 9, 2105-2116 (2016)

15. Almeida, R.: A Caputo fractional derivative of a function with respect to another function. Commun. Nonlinear Sci. Numer. Simul. 44, 460-481 (2017)

16. Abdo, M.S., Panchal, S.K., Saeed, A.M.: Fractional boundary value problem with $\psi$-Caputo fractional derivative. Proc. Indian Acad. Sci. Math. Sci. 129(5), 65 (2019)

17. Derbazi, Ch., Baitiche, Z., Benchohra, M., Cabada, A.: Initial value problem for nonlinear fractional differential equations with $\psi$-Caputo derivative via monotone iterative technique. Axioms 9, 57 (2020). https://doi.org/10.3390/axioms9020057

18. Samet, B., Aydi, H.: Lyapunov-type inequalities for an anti-periodic fractional boundary value problem involving $\psi$-Caputo fractional derivative. J. Inequal. Appl. 2018, 286 (2018)

19. Vivek, D., Elsayed, E.M., Kanagarajan, K.: Theory and analysis of partial differential equations with a $\psi$-Caputo fractional derivative. Rocky Mt. J. Math. 49(4), 1355-1370 (2019)

20. Rezapour, Sh., Ntouyas, S.K., Iqbal, M.Q., Hussain, A., Etemad, S., Tariboon, J.: An analytical survey on the solutions of the generalized double-order $\varphi$-integro-differential equation. J. Funct. Spaces 2021, Article ID 6667757 (2021)

21. Salim, A., Benchohra, M., Karapinar, E.: Existence and Ulam stability for impulsive generalized Hilfer-type fractional differential equations. Adv. Differ. Equ. 2020, 601 (2020)

22. Afshari, H., Karapinar, E.: A discussion on the existence of positive solutions of the boundary value problems via $\psi$-Hilfer fractional derivative on b-metric spaces. Adv. Differ. Equ., 2020, 616 (2020)

23. Tariboon, J., Ntouyas, S.K., Sudsutad, W.: Nonlocal Hadamard fractional integral conditions for nonlinear Riemann-Liouville fractional differential equations. Bound. Value Probl. 2014, 2014253 (2014). https://doi.org/10.1186/s13661-014-0253-9

24. Ntouyas, S.K., Tariboon, J., Sudsutad, W.: Boundary value problems for Riemann-Liouville fractional differential inclusions with nonlocal Hadamard fractional integral conditions. Mediterr. J. Math. 13, 939-954 (2016)

25. Samet, B., Vetro, C., Vetro, P.: Fixed point theorems for $\alpha-\psi$-contractive type mappings. Nonlinear Anal. 75 2154-2165 (2012)

26. Podlubny, l.: Fractional Differential Equations. Academic Press, San Diego (1999)

27. Samko, G., Kilbas, A., Marichev, O.: Fractional Integrals and Derivatives: Theory and Applications. Gordon \& Breach, New York (1993)

28. Kilbas, A.A., Srivastava, H.M., Trujillo, J.J.: Theory and Applications of Fractional Differential Equations. North-Holland Mathematics Studies, vol. 204. Elsevier, Amsterdam (2006)

29. Osler, T.J.: Leibniz rule for fractional derivatives generalized and an application to infinite series. SIAM J. Appl. Math. 18(3), 658-674 (1970)

30. Amini-Harandi, A.: Endpoints of set-valued contractions in metric spaces. Nonlinear Anal. 72, 132-134 (2010)

31. Karapinar, E., Samet, B.: Generalized $\alpha-\psi$-contractive type mappings and related fixed point theorems with applications. Abstr. Appl. Anal. 2012, Article ID 793486 (2012)

32. Karapinar, E., Kumam, P., Salimi, P.: On $\alpha-\psi$-Meir-Keeler contractive mappings. Fixed Point Theory Appl. 2013, 94 (2013)

33. Karapinar, E., Abdeljawad, T., Jarad, F.: Applying new fixed point theorems on fractional and ordinary differential equations. Adv. Differ. Equ. 2019, 421 (2019) 
34. Mohammadi, B., Rezapour, Sh., Shahzad, N.: Some results on fixed points of $\alpha-\psi$-Ciric generalized multifunctions. Fixed Point Theory Appl. 2013, 24 (2013)

35. Smart, D.R.: Fixed Point Theorems. Cambridge University Press, Cambridge (1980)

Submit your manuscript to a SpringerOpen ${ }^{\circ}$ journal and benefit from:

- Convenient online submission

$\checkmark$ Rigorous peer review

- Open access: articles freely available online

- High visibility within the field

Retaining the copyright to your article

Submit your next manuscript at $\gg$ springeropen.com 